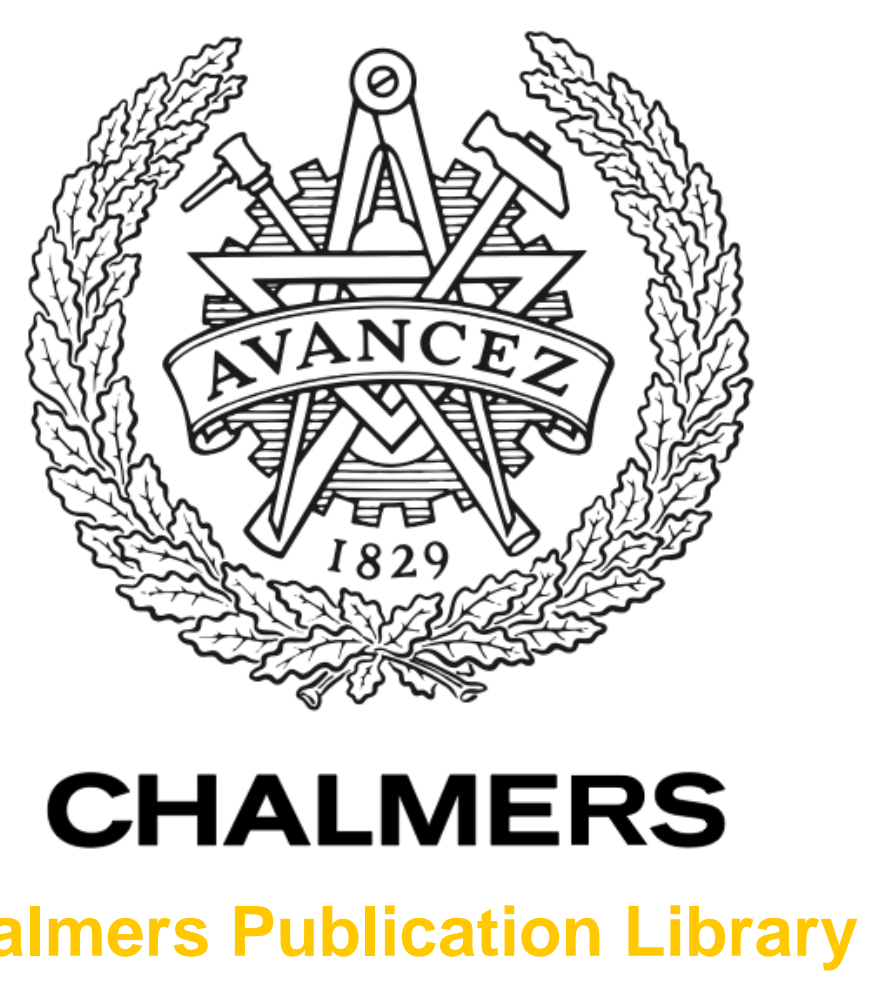

Chalmers Publication Library

\title{
Geometric division problems, quadratic equations, and recursive geometric algorithms in Mesopotamian mathematics
}

This document has been downloaded from Chalmers Publication Library (CPL). It is the author's version of a work that was accepted for publication in:

Archive for History of Exact Sciences (ISSN: 0003-9519)

Citation for the published paper:

Friberg, J. (2014) "Geometric division problems, quadratic equations, and recursive geometric algorithms in Mesopotamian mathematics". Archive for History of Exact Sciences, vol. 68(1), pp. 1-34.

http://dx.doi.org/10.1007/s00407-013-0122-4

Downloaded from: http://publications.lib.chalmers.se/publication/193131

Notice: Changes introduced as a result of publishing processes such as copy-editing and formatting may not be reflected in this document. For a definitive version of this work, please refer to the published source. Please note that access to the published version might require a subscription. 


\title{
Geometric Division Problems, Quadratic Equations, and Recursive Geometric Algorithms in Mesopotamian Mathematics
}

\author{
Jöran Friberg
}

\begin{abstract}
Most of what is told in this paper has been told before by the same author, in a number of publications of various kinds, but this is the first time that all this material has been brought together and treated in a uniform way. Smaller errors in the earlier publications are corrected here without comment.

It has been known since the 1920's that quadratic equations played a prominent role in Babylonian mathematics. See, most recently, Høyrup 1996 and 2002. What has not been known, however, is how quadratic equations came to play that role, since it is difficult to think of any practical use for quadratic equations in the life and work of a Babylonian scribe. One goal of the present paper is to show how the need to find solutions to quadratic equations actually arose in Mesopotamia not later than in the second half of the 3rd millennium $\mathrm{BC}$, and probably before that, in connection with certain geometric division of property problems. This issue was brought up for the first time in Friberg 2009.

In this connection, it is argued that the tool used for the first exact solution of a quadratic equation was either a clever use of the "conjugate rule" or a "completion of the square", but that both methods ultimately depend on a certain division of a square, the same in both cases.

Another, closely related goal of the paper is to discuss briefly certain of the most impressive achievements of anonymous Babylonian mathematicians in the first half of the 2nd millennium BC, namely recursive geometric algorithms for the solution of various problems related to division of figures, more specifically trapezoidal fields. For an earlier, comprehensive (but less accessible) treatment of these issues, see Friberg 2007b, Ch. 11 and App. 1.
\end{abstract}

\section{Metro-Mathematical School Texts from the Proto-Literate Period in Mesopotamia (around $3000 \mathrm{BC}$ )}

1.1. For a long time before the invention of writing, probably for several millennia, counting and recording were performed in vast regions of the Middle East by use of small "pre-literate number tokens" of clay in simple geometric forms (cylinders, spheres, cones, pyramids) with a system of assigned metrological-numerical values. (See Friberg 2007a, App. 4.10, and also Friberg 1999.) The oldest known inscribed clay tablets are from the "proto-literate period" in Mesopotamia, slightly before and around $3000 \mathrm{BC}$, and are written in the "proto-cuneiform" script, a precursor of the more well known Sumerian/Babylonian cuneiform script. Already in these earliest written documents, systems of measures and measure notations for lengths and surfaces, capacities of containers, weights, and parts of the year, were extremely well developed, and beautifully coordinated with a system of sexagesimal counting numbers in non-positional notation. (See Friberg op. cit., App. 4.1-2, 4.4-6, 4.9). There were different number signs for 1(60), for 10(60), for 1(60 - 60), for 10(60 - 60), etc. Lengths were probably, as later in the Sumerian period, expressed in terms of "rods" $(6 \mathrm{~m}$.) and "ropes" (= 10 rods), etc. Surfaces were, correspondingly, measured in terms of square rods (in Sumerian sar) and square ropes (in Sumerian iku), in other words in "quadratic area measure". This is the only textual indication that the notion of a "square" was known already in this remote period. (Presumably, it would not be difficult to find non-textual, concrete evidence in architecture, art, etc., that the notion of a square was well known also in remote times in Mesopotamia.)

Only a handful of suspected mathematical school texts from the proto-literate period are known. Just like practically all known mathematical texts from the third millennium BC, they should rather be called "metro-mathematical", because it is evident that their purpose was just as much training in "metrology", that is in the use of the various systems of measures and measure notations, as training in "mathematics", that is in counting with sexagesimal numbers and performing various kinds of computations.

The clearest example of a proto-literate metro-mathematical text is they clay tablet W 19408, 76 (Nissen, Damerow, and Englund 1993, Fig. 50), on which are given the side lengths of two quadrilateral fields (by which is meant more or less rectangular fields with four straight sides). The text is probably an assignment, in the sense that a young student was supposed to compute the areas of the two fields. If the two areas are computed by use of a certain inexact "quadrilateral area rule", 
namely in each case as the half-sum of the two lengths "along" times the half-sum of the two lengths "across", it turns out that they can both be expressed as very large and very round area numbers. Obviously, the data must have been chosen carefully and deliberately by a teacher (the author of the exercise) to produce this kind of nice numbers. It is, by the way, common in the study of Mesopotamian mathematics that particularly interesting results are obtained by trying to understand how teachers constructing mathematical exercises for their students cleverly fabricated the initial data for those exercises.

1.2. Other known examples of proto-literate mathematical texts are supposed applications of a certain, quite elegant, "field expansion procedure". (See Friberg 2007a, sections $1.1 \mathrm{~d}$ and $8.1 \mathrm{~b}-\mathrm{c}$, and Friberg 1997/98, sections 2-4.) Take, for instance, W 14148 (Friberg 1997/98, 9; Fig. 1.1 below), originally published in Green and Nissen 1987, ATU 2, pl. 59 :

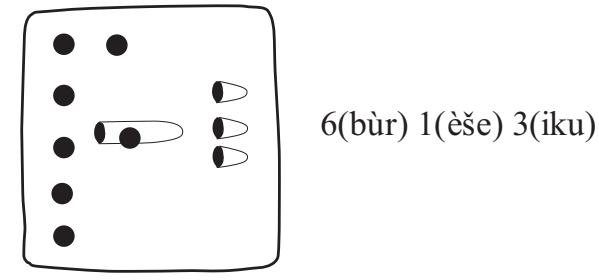

Fig. 1.1. W 14148. An area produced by the field expansion procedure

This is a small text from Warka, ancient Uruk, where a large number of proto-literate clay tablets have been found. It is a note about an area written with the special proto-cuneiform area number signs for 1 (bùr) $=18 \mathrm{iku}$, for 1 (èše) $=6 \mathrm{iku}$, and for 1 (iku). (A notation like 6 (bùr) stands for the special area number sign 1(bùr) repeated 6 times, etc.) The area in question is

$$
6 \text { bùr } 1 \text { èše } 3 \mathrm{iku}=191 / 2 \text { èše = 1(60) } 57 \mathrm{iku}(=117 \mathrm{iku}) \text {. }
$$

Note: A notation like 1(60) 57 is used here for a sexagesimal number in non-positional notation. The corresponding notation for a sexagesimal number in positional or place value notation would be simply 157 .

This area is typical for areas computed by use of the field expansion procedure. Indeed, note that

a) $191 / 2$ èše $=20$ èše $-1 / 40$ of 20 èše $=$ approximately 20 èše $(=12,000$ square rods $)$.

b) $1(60) 57 \mathrm{iku}=13 \cdot 9 \mathrm{iku}=(11 / 12) \cdot(11 / 8) \cdot 12 \cdot 8 \mathrm{iku}$.

This means that the area number inscribed on W 14148 is a) very close to a conspicuously "round" area number, and b) equal to the area of a rectangular field with the side along (1 1/12) $\cdot 12$ ropes and the side across $(11 / 8) \cdot 8$ ropes. A rectangular field like this is the result of a slight expansion in two directions of a rectangular field with its sides in a simple ratio $(12: 8=3: 2)$.

Further explicit examples of suspected school texts related to the field expansion procedure will be given in sections 2.1 and 3.1 below. There it will also be shown that the field expansion procedure may have been a clever method of solving certain problems involving quadratic equations by means of iterated approximations.

1.3. In a proto-literate "field-division text" MSVO 1, 2 (Englund and Grégoire 1991; Friberg 1997/98, 19), the areas are computed for five rectangular fields of various sizes, belonging to five named persons. The sum of the five areas, which is recorded on the reverse of the clay tablet, is

$$
3 \text { šàr } 52 \text { bùr } 2 \text { èše } 5 \mathrm{iku}=1(60 \cdot 60) 9(60) 53 \mathrm{iku}=1(60 \cdot 60) 10(60)-7 \mathrm{iku} \quad(1 \text { šàr }=60 \text { bùr })
$$

In other words, the sum of the five areas is

$7 \cdot 10 \cdot 60 \mathrm{iku}-7 \mathrm{iku}=7 \cdot 10 \cdot 60 \mathrm{iku}$ (square ropes) minus precisely $1 / 600$ of that round number.

This nearly round area number is hardly accidental, it must have been the result of a difficult and laborious computation.

Another suspected example of a school text from the proto-literate period in Mesopotamia is the "bread-and-beer text" MSVO 4, 66 (Englund 1996; Friberg 1999a, 112-115; Friberg 2005, 62-65; and Fig. 1.2 below.) This text begins with an account of 
$60+2 \cdot 60+2 \cdot 60+(2 \cdot 260+60)+5 \cdot 2 \cdot 60+5 \cdot 20 \cdot 60=20 \cdot 60+5 \cdot 20 \cdot 60(=7,200)$ bread rations of six different sizes, with a corresponding cost in flour of $1 \mathrm{M}$ (a certain proto-literate unit of capacity measure, possibly about 5 liters), $1 / 2 \mathrm{M}, 1 / 3 \mathrm{M}, 1 / 4 \mathrm{M}, 1 / 5 \mathrm{M}$, and $1 / 6 \mathrm{M}$, respectively. There are relatively small numbers of rations of the larger sizes, but $5 \cdot 20 \cdot 60=6,000$ rations of the smallest size. The total cost in flour for all the bread rations is nearly $221 / 2 \cdot 60 \mathrm{M}$. Next there is an account of the cost in flour for various amounts of three categories of beer. The total cost in flour for all the beer (together $10 \cdot 60=600$ jars) is nearly $71 / 2 \cdot 60 \mathrm{M}$. Recorded on the reverse of the clay tablet is the total cost in flour for both bread and beer, which is

$900 \mathrm{M}+2 \cdot 300 \mathrm{M}+9 \cdot 30 \mathrm{M}+4 \cdot 5 \mathrm{M}+1 \mathrm{M}=1791 \mathrm{M}$

$=2 \cdot 900 \mathrm{M}$ minus precisely $1 / 200$ of that round number.

This nearly exact result is hardly accidental, it must have been the result of another difficult and laborious computation.

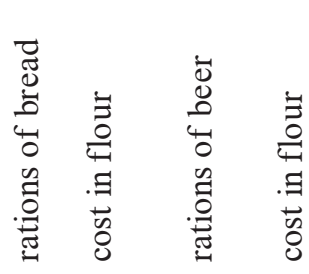

$o b v$.

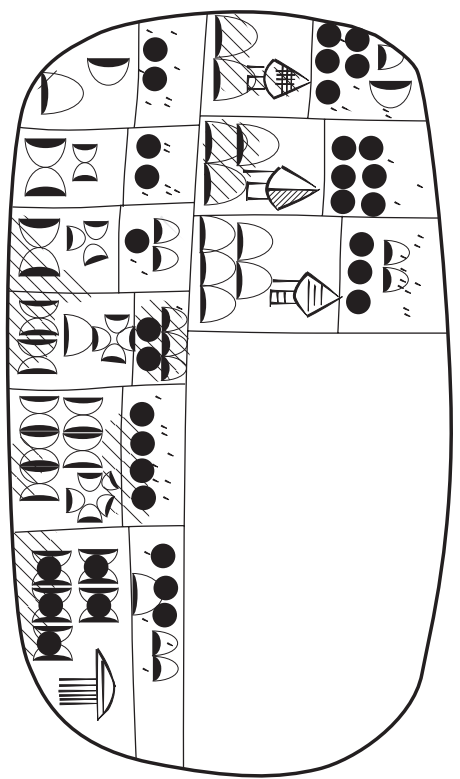

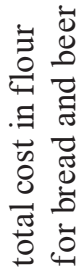

rev.

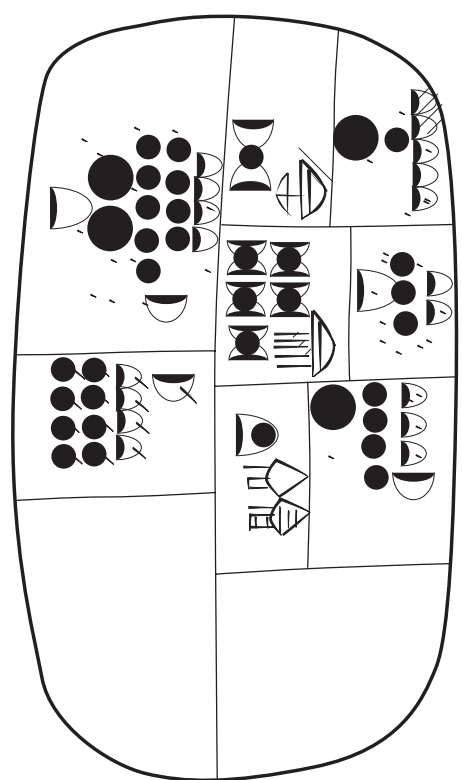

Fig. 1.2. MSVO 4, 66. A proto-literate school text: A summation of rations of bread and beer

The mentioned examples show that there can be no doubt that learning to divide given parcels of land into shares according to some intricate set of rules, or dividing given amounts of food stuff into rations of various sizes according to some other intricate set of rules, must have been an important part of the metro-mathematical education given to the young scribes in the scribe schools that must have existed in one or several Mesopotamian large urban areas already in the proto-literate period 5000 years ago.

\section{Metro-Mathematical School Texts from the Early Dynastic III Period (around $2500 \mathrm{BC}$ )}

2.1. In the Old Sumerian Early Dynastic III period, Mesopotamian mathematics had taken important strides forward. Yet, a connection was still maintained with the mathematical ideas of the preceding proto-literate period, as is shown by the inscriptions in the two columns of the Early 
Dynastic clay tablet TSS 926 shown in Fig. 2.1 below (Jestin 1937; Friberg 2007a, 147). In each column, the sides of a quadrilateral field are given, expressed in terms of sexagesimal numbers in nonpositional notation. The inscription in the well preserved first column is transliterated (in Sumerian) and translated (in English) below, to the right of the copy of the clay tablet.

Like its proto-literate predecessor, mentioned above, this mathematical text is probably an assignment, in the sense that a young student was supposed to compute the areas of the two fields.

It is easy to see that the half-sums of the lengths of the sides along and the sides across (recorded in the first column of the text) would be, respectively,

$$
(120+125) / 2 \text { rods }=1221 / 2 \text { rods }=121 / 4 \text { ropes, and }
$$

$(60+70) / 2$ rods $=65$ rods $=61 / 2$ ropes.

Correspondingly, the computed area of the quadrilateral would be of the following form:

$121 / 4$ ropes $\cdot 61 / 2$ ropes $=791 / 21 / 8 \mathrm{iku}$ (square ropes) $=$ almost precisely $80 \mathrm{iku}$ (= 4 bùr 1 èše $2 \mathrm{iku}$ ).

Alternatively, the area of the quadrilateral can be expressed in the following way:

$(11 / 48 \cdot 12$ ropes $) \cdot(11 / 12 \cdot 6$ ropes $)$.

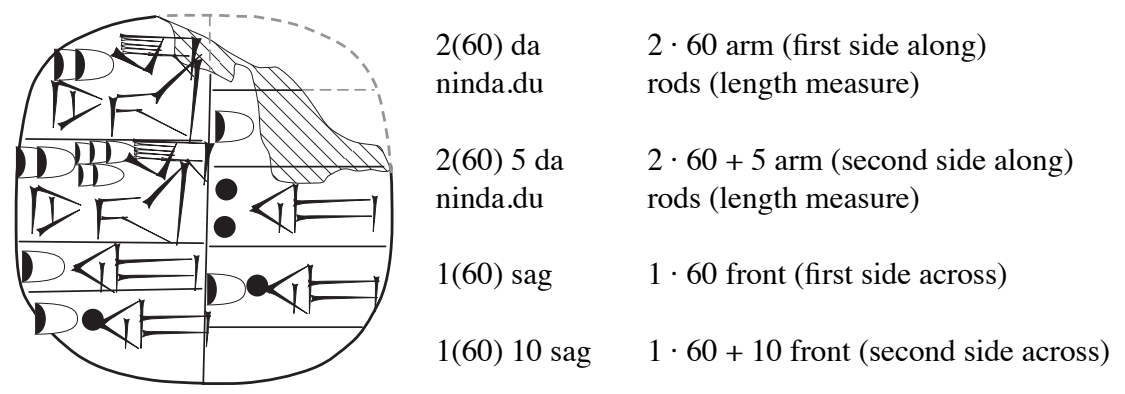

Fig. 2.1. TSS 926. An example of data produced by the field expansion procedure

As will be shown in connection with a similar example in section 3.1 below, whenever the computed area of a quadrilateral in a Mesopotamian (Sumerian or Babylonian) mathematical exercise can be expressed both as a (nearly) round number and as a round number multiplied by two factors of the form $11 / n$, where $n$ is a "regular" sexagesimal number (more about this later), then the exercise can be suspected of being the result of the application of the mentioned, initially proto-literate, field expansion procedure. The purpose of that procedure seems, in each case, to have been to compute the sides of a quadrilateral field with (approximately) a given area and a given relation between the halfsides along and the half-sides across!

2.2. The Early Dynastic III tablet TSS188 (Jestin 1937; Friberg 2007a, 148) contains the earliest known explicit reference to a square. In the copy of the clay tablet in Fig. 2.2 below, it is the diamondshaped cuneiform logographic sign to the right in the first line of the text, normally with the meaning "(to be) equal", but here with the more precise meaning "a figure with equal sides (and right angles)".

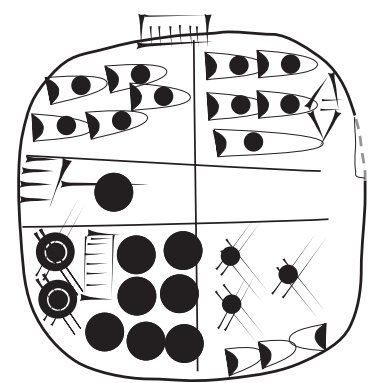

\author{
aša \\ 50(60) 50(60) sá \\ gal $\times 1(60$ bùr $)(=1(60 \cdot 60$ bùr $))$ \\ 27(60 bùr) 30(bùr)
}

$21 / 2$ field measure

$50 \cdot 60$ (ninda) $50 \cdot 60$ (ninda) equal

$1(60 \cdot 60)$ bùr $(=3,600$ bùr $)$

27(60) 30 bùr (= 1,650 bùr $)$

$21 / 2$

Fig. 2.2. TSS 188. The area of an unrealistically large square 
In this text, the area of a very large square with the side $50(60)$ rods $(=3,000$ rods $=1,800$ meters!) has been computed. The result is expressed in terms of the Sumerian large area measure bùr $=30(60)$ sq. rods and special Sumerian number signs for area measures. Interestingly, the stated answer is not correct.

Apparently, the computation (which is not shown in the text) was intended to proceed in a quite clever way, namely as follows:

$$
\begin{aligned}
& \text { sq. }(50(60) \text { rods })=\text { sq. } 5 \cdot \text { sq. } 10 \cdot \text { sq. }(60 \text { rods })=25 \cdot 1(60) 40 \cdot 2 \text { bùr }= \\
& 25 \cdot 3(60) 20 \text { bùr }=21 / 2 \cdot 33(60) 20 \text { bùr }=1(60 \cdot 60) 23(60) 20 \text { bùr. }
\end{aligned}
$$

Instead, however, a simple numerical error in the middle of the computation lead to the following incorrect result (the one inscribed in the lower half of the clay tablet):

$$
\begin{aligned}
& \text { sq. }(50(60) \text { rods })=\text { sq. } 5 \cdot \text { sq. } 10 \cdot \text { sq. }(60 \text { rods })=25 \cdot 1(60) 40 \cdot 2 \text { bùr }= \\
& 25 \cdot 3(60) \underline{30} \text { bùr }=21 / 2 \cdot \underline{35}(\underline{60}) \text { bùr }=1(60 \cdot 60) \underline{27}(\underline{60} \underline{30} \text { bùr. }
\end{aligned}
$$

Note: The abbreviation sq. stands here for 'square', in the geometric sense, as an area. The concept of a number in the second power was not known in ancient Mesopotamia, at least not in the 3rd millennium BC. The notation $a \cdot b$ stands, indiscriminately, for either the area of a rectangle with sides of lengths $a$ and $b$, or for $b$ (an area, a length, or a number) multiplied by the number $a$.

2.3. Also from the Early Dynastic III period are three known metro-mathematical tables of squares or, more precisely, "tables of areas of square fields". One of these tables (VAT 12593) lists the areas of a decreasing series of squares with the sides 10(60) rods, 9(60) rods, and so on (Deimel 1923; Friberg 2007a, 149). This is the oldest known metro-mathematical table text.

A somewhat younger, but even larger and much more elaborate table text (CUNES 50-08-001) begins by listing the areas of an increasing series of squares, with sides progressing in regular steps from 1 rod to $10(60 \cdot 60)$ rods. Then follow several other tables of similar types, ending with a table for areas of of a series of squares with sides as small as 1/24 rod (Friberg op. cit., App. 7).

Metro-mathematical tables of areas of square fields of the mentioned kind are, in a way, predecessors of both Old Babylonian tables of squares and Old Babylonian metrological tables.

An interesting deviation from this pronounced interest in the areas of squares is another table text from the Early Dynastic III period (MS 3047, obv.; Friberg op. cit., 150; and Fig. 2.3 below). It is a curious table of areas of a series of six rectangles, all (unrealistically) with the length 60 times as long as the width. The text ends by mentioning the sum of the six computed areas, so it is a playful imitation of a field-division text like the proto-literate text MSVO 1,2 mentioned in section 1.2 above.
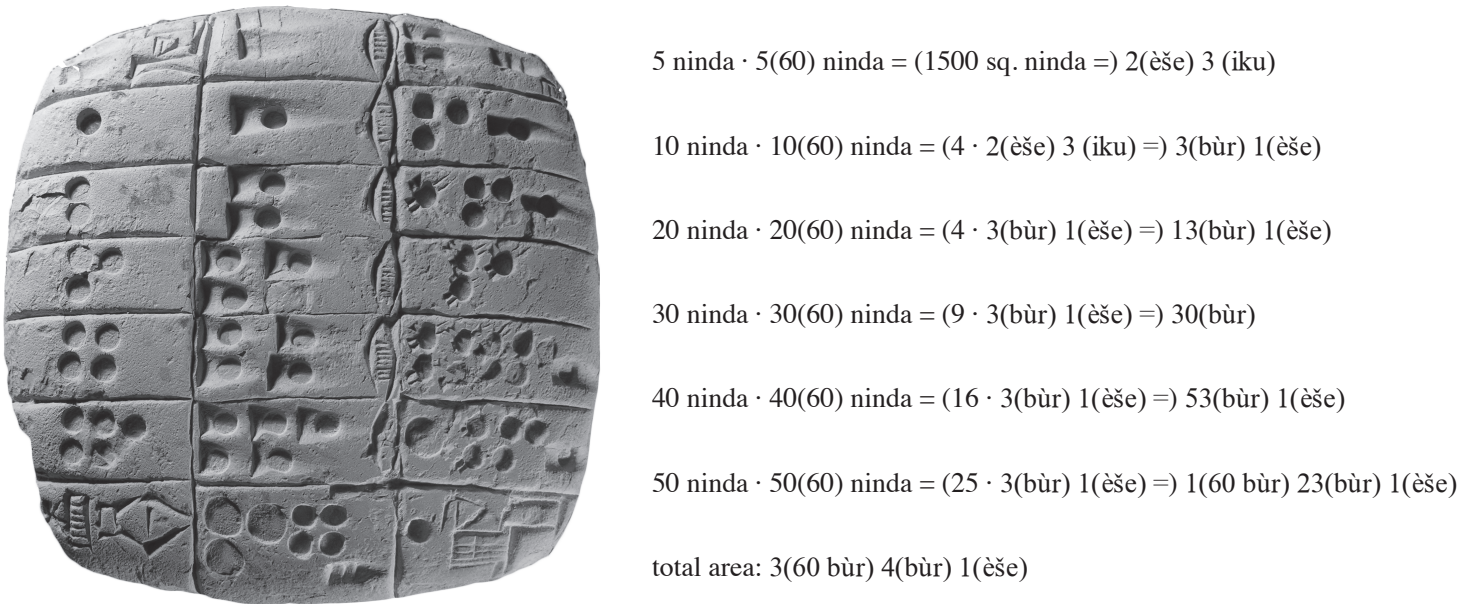

Fig. 2.3. MS 3047. A summation of the areas of six very long and narrow rectangles 
The tendency of mathematical exercises to deal with unrealistically large numbers (as in W 19408, 76, MSVO 4, 66, TSS 188, and MS 3047 above) can be observed also in a couple of well known Early Dynastic III "metric division exercises" (TSS 50 and TSS 671; Jestin 1937; Høyrup 1982; Friberg op. cit., 414). There the content of a granary, 5(60 -60 -60) 20(60 - 60) sìla (the sila was a capacity measure, equal to approximately 1 liter) $=1,152,000$ sìla, is to be divided into rations of 7 sila each. Since 7 is not a divisor of 60, the division must have been accomplished by use of an elaborate metric division algorithm, as in a parallel and roughly contemporary metro-mathematical text from Ebla, a city state in modern Syria not far from northern Mesopotamia (TM.75.G 1392; Friberg 1986).

\section{Metro-Mathematical School Texts from the Old Akkadian Period (c. 2340-2200 BC)}

3.1. The text shown in Fig. 3.1 below (Luckenbill 1930; Friberg 2007a, 402) is from the Old Akkadian period. It specifies the sides and the area of a long and narrow trapezoidal field. The given unequal widths are 1(60) 10 rods and $441 / 2$ rods (half-sum $571 / 4$ rods), and the length is 5(60) 15 rods. The area of the field is claimed to be 10 bùr $=5(60 \cdot 60)$ square rods, a large and round area number. The last two lines of the text give the name of the writer, 'The-king-is-my-wall', and the name of the field, 'The-field-by-the-large-and-grand-marsh', which sound like fictive names.

Since the area of the field is a round number while its length can be written as $11 / 6 \cdot 4(60) 30$, this seems to be the result of yet another application of the mentioned proto-literate field expansion procedure.

$\begin{array}{ll}\text { 1(60) } 10 \text { sag } 441 / 2 & \text { 1(60) } 10 \text { front (= width) } 441 / 2 \\ 5(60) 15 \text { both lengths } \\ \text { 5(60) } 15 \text { uš hi.a }\end{array}$

Fig. 3.1. A 786. Another example of data produced by the field expansion procedure

The construction of the data can be explained as follows: The author of the text wanted to construct a trapezoid with the round area 10 bùr $=5(60 \cdot 60)$ square rods, and with the ratio of the length to the half-sum of the widths close to $5: 1$. For that purpose, he started with a rectangle with

the initial length $4(60) 30$ rods, and the initial width $1 / 5 \cdot 4(60) 30$ rods $=54$ rods

step 1

The corresponding initial area could then be shown to be 4(60) 30 rods $\cdot 54$ rods $=4(60 \cdot 60) 3(60)$ sq. rods $=8$ bùr 1 iku 80 sar, nearly $1(60 \cdot 60)$ sq. rods $=2$ bùr less than the wanted area. In other words, the area deficit was close to $1 / 4$ of the initial area. To substantially reduce this deficit, the author of the text extended the initial length by $1 / 6$, and obtained

$$
\text { the extended length }=11 / 6 \cdot 4(60) 30 \text { rods }=5(60) 15 \text { rods }
$$

step 2

The resulting extended area was

$$
11 / 6 \cdot 4(60 \cdot 60) 3(60) \text { sq. rods }=4(60 \cdot 60) 3(60) \text { sq. rods }+40(60) 30 \text { sq. rods }=4(60 \cdot 60) 43(60) 30 \text { sq. rods. }
$$

In Sumerian area measure, 9 bùr $8 \mathrm{iku} 10$ sar. The new area deficit was then 16(60) 30 sq. rods, slightly less than $1 / 16$ of the new area. To eliminate most of this new deficit, the author of the text extended the width by a factor of $1 /(162 / 3)=3 / 6036 /(60 \cdot 60)$, obtaining

$$
\text { the extended width }=(1+3 / 6036 /(60 \cdot 60)) \cdot 54 \mathrm{rods}=5714 / 6024 /(60 \cdot 60) \mathrm{rods}=\text { appr } 571 / 4 \mathrm{rods}
$$

The author of the text probably did not bother to compute the area of the twice extended 
rectangle, but instead immediately declared it to be 10 bùr $=5(60 \cdot 60)$ square rods. (The exact result is $5(60 \cdot 60) 331 / 4$ square rods, or about $5(60 \cdot 60)$ square rods $+1 / 600$ of that number.) Moreover, since he wanted the final result to be a trapezoid rather than a rectangle, he chose to let the upper width be 1(60) 10 rods. It followed that the lower width had to be $441 / 2$ rods, which is the value given in the text.

1.

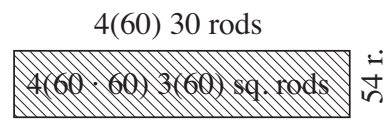

3.

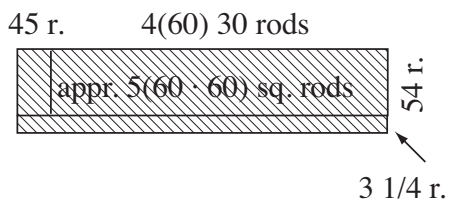

2.

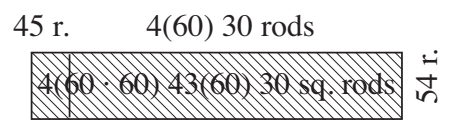

4.

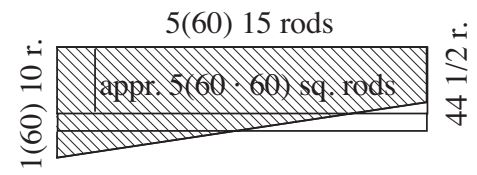

Fig. 3.2. How the (proto-literate) field expansion procedure operates

The appearance of the number $162 / 3$ in this reconstructed application of the field extension procedure is, possibly, the earliest known appearance in a Mesopotamian mathematical text of a deliberately chosen "regular sexagesimal number". A regular sexagesimal number is a sexagesimal number $n$ which divides some power of the base 60 , and which therefore has another, finitely expressible sexagesimal number (say, an integer) as its "reciprocal". In this particular case, 16 2/3 has the reciprocal 3(60) 36 because $162 / 3 \cdot 3(60) 36=1(60 \cdot 60)$. -- Note that this surmised example of the use of a regular sexagesimal number in a computation unexpectedly appears in a cuneiform text from a period well before the invention of sexagesimal numbers in place value notation!

The frequent appearance of results of applications of the (proto-literate) field extension procedure in Mesopotamian metro-mathematical texts from the 3rd millennium BC is potentially historically important for the following reason: Apparently, the primary purpose of the field extension procedure was (for some unknown reason) to construct a rectangular field of given size and form, in other words a rectangle with a given area and a given ratio of length and width. In modern notations, this is a pair of equations of the type

$$
l \cdot w=A, \quad l / w=f .
$$

A mathematically equivalent "quadratic-linear" pair of equations is, of course,

$$
\text { sq. } l=f \cdot A, \quad w=l l f \text {. }
$$

Therefore, the field extension procedure can be interpreted as a primitive but clever way of solving geometrically a quadratic problem of the very simplest kind, indirectly involving a square side computation, by a kind of successive approximations. (For a detailed discussion of various clever methods for square side computations, by use of successive approximations, in Old and Late Babylonian mathematical texts, the interested reader is referred to Friberg 2007b, Ch. 16.7.)

3.2. Several known small school texts from the Old Akkadian period are witnesses of a rising interest in "metric algebra", by which is meant here finding solutions to geometric problems by skillful manipulations of rectangles and squares, with sizes of sides and surfaces expressed in terms of current length and area measures. (See Friberg 2007a, 403-409). Some of the texts are examples of "metric division" where the area and the length of one side of a rectangle are known, while the length of the second side has to be computed. In one such case, the area of a rectangle is 1 square rope, while the length of the known side is 4(60) 3 rods. Typically, 4(60) $3=3^{5}$ is a regular sexagesimal number. Some other texts are examples of "metric squaring", typically exercises in the use of the "binomial rule" 
sq. $(p+q)=$ sq. $p+$ sq. $q+2 p \cdot q$,

since the given length of a side is the sum of a large number and a much smaller number. This is interesting, because the inverse "square side" problem, that of finding the length of the side of a square of given area (in modern abstract terms, computing a square root), can be solved by use of the binomial rule, together with successive approximations.

3.3. On the Old Akkadian school text IM 58045 (Friberg 2007a, 409), shown in Fig. 3.3 below with a translated cuneiform inscription, there is a picture of a trapezoid with parallel sides of 3 reeds -1 cubit $=17$ cubits and 1 reed 1 cubit $=7$ cubits, and with a height of 2 reeds $=12$ cubits $=1$ rod. An easy computation shows that the area of the trapezoid is precisely 12 cubits $\cdot 12$ cubits $=1$ sq. rod, a round (but surprisingly small) area number. A transversal line divides the trapezoid into two subtrapezoids. It is likely that it was a student's task to find the length of the transversal line in the case when it bisects the trapezoid, that is, when the areas of the two sub-trapezoids are equal.

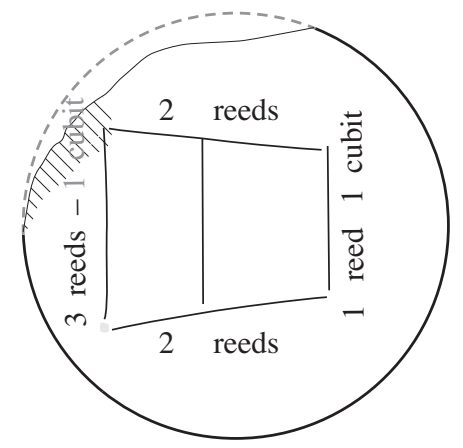

Fig. 3.3. IM 58045. The data for a trapezoid division problem

There is no direct indication how the student would have attacked this problem. Essentially, there are two different methods the student could conceivably have used, if he did not know the answer beforehand. (An uninteresting and trivial possibility is that the student already was familiar with the "trapezoid bisection equation", discussed below, but then the question remains how that equation had been discovered in the first hand.) The first of these two methods will be considered in the present section of this paper. Indeed, consider (as in Fig. 3.4 below) a trapezoid with parallel sides of given lengths $a$ and $b$, with $b>a$, and a given height $h$. Then the area $A$ and the "growth rate" $f=(b-a) / h$ can be computed. Imagine that the trapezoid is divided by a transversal into two sub-trapezoids, one of area $P$, the other of area $Q$, as in Fig. 3.4. Let the unknown length of the transversal be $d$, and let the unknown distance between the transversal and the longer parallel be $u$.
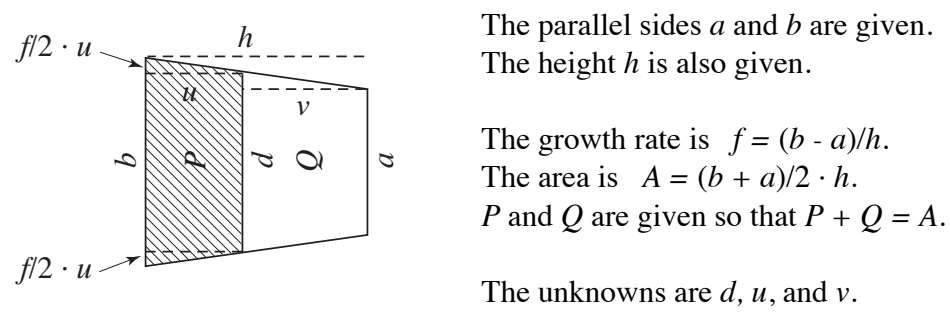

The unknowns are $d, u$, and $v$.

\section{Fig. 3.4. A possible setup for a trapezoid division problem}

Now, just as for the whole trapezoid $(b+a) / 2 \cdot h=A$ and $b-a=f \cdot h$, so for the upper subtrapezoid

$$
(b+d) / 2 \cdot u=P, \quad b-d=f \cdot u .
$$

The unknowns $d$ and $u$ can then be computed as the solutions to the following "rectangular-linear system of equations": 
$(b+d) / 2 \cdot u=P, \quad b-d=f \cdot u, \quad$ where $b, f$, and $P$ are known.

Therefore, an application of the "conjugate rule"

$(b+d) \cdot(b-d)=$ sq. $b-$ sq. $d$

shows that, alternatively, $d$ and $u$ can be found as the solutions to the following "quadratic-linear system of equations":

sq. $b-$ sq. $d=f \cdot 2 P, \quad b-d=f \cdot u, \quad$ where $b, f$, and $P$ are known.

After a slight rearrangement of the terms in the two equations, one gets instead

sq. $d=$ sq. $b-f \cdot 2 P, \quad u=(b-d) / f, \quad$ where $b, f$, and $P$ are known.

This means that if the Old Akkadian student knew the conjugate rule and if he knew how to compute a square side, then he could find first the value of $d$, and thereafter also the value of $u$.

In the Old Akkadian school text IM 58045 considered above, the given values are

$b=17$ cubits, $a=7$ cubits, $h=12$ cubits, and (probably) $P=Q=A / 2$.

Consequently

$2 P=A=$ sq. $(12$ cubits $)=2(60) 24$ sq. cubits, $f=(17-7) / 12=5 / 6$.

In view of what was shown above, this means that $d$ can be computed as the following square side (abbreviated sqs.):

$$
\begin{aligned}
& d=\text { sqs. (sq. } b-f \cdot 2 P)=\text { sqs. (sq. }(17 \text { cubits })-5 / 6 \cdot 2(60) 24 \text { sq. cubits })= \\
& \text { sqs. }((4(60) 49-2(60)) \text { sq. cubits })=\text { sqs. }(2(60) 49 \text { sq. cubits })=13 \text { cubits. }
\end{aligned}
$$

Then also

$u=(17$ cubits -7 cubits $) / 2=5$ cubits.

Note that the teacher had chosen the data of the exercise so that, in this particular case, the computation of the square side was easy, because 2(60) 49 sq. cubits is precisely the square of 13 cubits.

Similarly, considering in Fig. 3.4 the lower sub-trapezoid with the area $Q$, it can be shown that sq. $d-$ sq. $a=f \cdot 2 Q$.

Therefore, in the special case when $P=Q$, so that the transversal bisects the trapezoid, it follows that

$$
\text { sq. } b-\mathrm{sq} . d=\mathrm{sq} . d-\mathrm{sq} . a \quad \text { or } \quad \text { (sq. } a+\mathrm{sq} \cdot b)=2 \text { sq. } d \text {. }
$$

When $a$ and $b$ are known, this is a quadratic equation for $d$, which may be called the "trapezoid bisection equation". When $a$ and $b$ are not known, the trapezoid bisection equation is instead an indeterminate quadratic equation for the triple $a, b, c$.

It is possible that a solution in integers to the indeterminate trapezoid bisection equation was found initially as a result of a fortuitous observation of the curious fact that

$$
\text { sq. }(7 \text { cubits })=49 \text { sq. cubits, } \quad \text { sq. }(13 \text { cubits })=2(60) 49 \text { sq. cubits, } \quad \text { sq. }(17 \text { cubits })=4(60) 49 \text { sq. cubits. }
$$

Admittedly, it may be only a reasonable conjecture that Old Akkadian students knew how the trapezoid transversal $d$ in Fig. 3.4 above can be computed by use of the quadratic equation

sq. $d=$ sq. $b-f \cdot 2 P, \quad$ where $b, f$, and $P$ are known.

However, it is a fact that Old Babylonian students knew how to use the very same quadratic equation for a similar purpose. This is shown, quite explicitly, by the solution procedure in the interesting "breach repair problem" in $\S 1$ of the Old Babylonian mathematical cuneiform text MS 3052 (Friberg 2007a, Fig. 10.2.4).

3.4. The second of the two methods which an Old Akkadian student may conceivably have used in order to compute the length of the transversal bisecting the trapezoid depicted on the tablet IM 58045 in Fig. 3.3 above (without already being familiar with the trapezoid bisection equation), proceeds as follows: It is geometrically obvious (see Fig. 3.4) that

$$
b \cdot u-f / 2 \cdot \text { sq. } u=P, \quad \text { where } b, f \text {, and } P \text { are known. }
$$


This is a quadratic equation for the unknown $u$, of a kind that was solved routinely in Old Babylonian mathematical problem texts, apparently through an application of a geometric version of the method called (by us) "completion of the square".

Therefore, if an Old Akkadian student knew how to apply a completion of the square, and if he also knew how to compute a square side, he could use the quadratic equation mentioned above in order to find the lengths of the trapezoid transversals in Figs. 3.3-4.

It is, by the way, important to understand that for an Old Akkadian student of mathematics, what we call the "binomial rule", the "conjugate rule", and "completion of the square" must all have been understood in their original, geometric sense. Indeed, in the Old Akkadian period, and even much later, abstract numbers and symbolic notations for objects or algebraic operations, etc., had not yet been invented. (For a detailed elaboration of remarks like these, interested readers are referred to Høyrup's LWS (2002). Høyrup was, indeed, the first one to understand that Old Babylonian mathematicians both formulated and solved quadratic equations and problems geometrically.)

Moreover, the word "rule" is more suitable here than the obviously anachronistic word "theorem", even if it is likely that Old Akkadian teachers used some kind of geometric arguments to convince their students about the validity of the rules. The forms that such geometric arguments could take (hypothetically) is shown below, in the diagrams in Figs. 3.5-6.

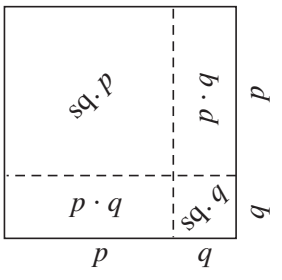

sq. $(p+q)=$

sq. $p+$ sq. $q+2 p \cdot q$

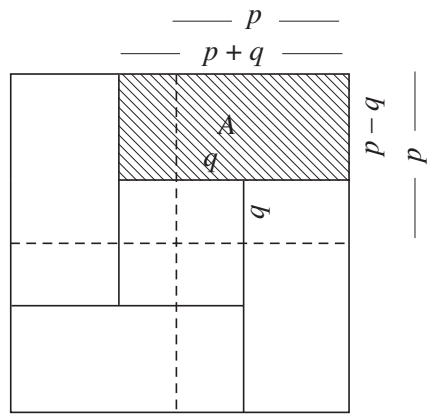

$(p+q) \cdot(p-q)=A$

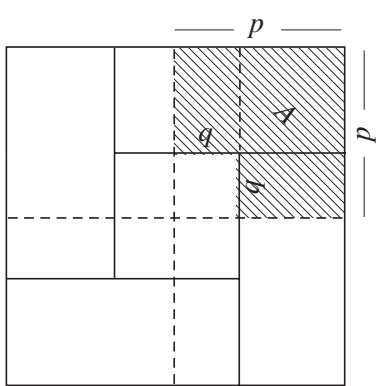

sq. $p-$ sq. $q=A$

$(p+q) \cdot(p-q)=$ sq. $p-$ sq. $q$

Fig. 3.5. Geometric demonstrations of the binomial and conjugate rules

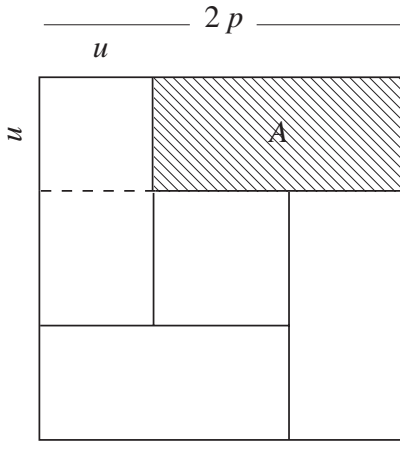

$2 p \cdot u-$ sq. $u=A$

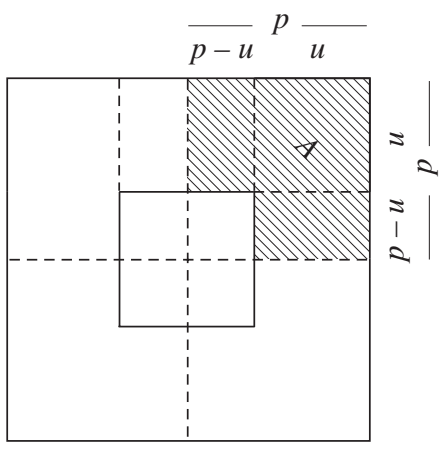

sq. $(p-u)=$ sq. $p-A$

Fig. 3.6. A geometric demonstration of the completion of the square

Note that no Mesopotamian clay tablets with diagrams of this kind are known to exist. See, however, the detailed discussion of related issues in Friberg 2007b, Ch. 1, which compares proofs in Euclid's Elements II with what is known about Babylonian "metric algebra". It is interesting to notice that the suggested demonstration of the conjugate rule is based on the same geometric configuration as the suggested demonstration of the completion of the square. In view of this intimate connection between the conjugate rule and the completion of the square, it is easy to understand why, as above, 
the following rectangular-linear system of equations for the unknowns $u$ and $d$

$$
(b+d) / 2 \cdot u=P, \quad b-d=f \cdot u, \quad \text { where } b, f \text {, and } P \text { are known }
$$

can be solved by use of the conjugate rule, while the alternative, the quadratic-linear system of equations

$$
b \cdot u-f / 2 \cdot \text { sq. } u=P, \quad b-d=f \cdot u, \quad \text { where } b, f \text {, and } P \text { are known }
$$

can be solved by use of a completion of the square.

\section{Metro-Mathematical School Texts from the Neo-Sumerian Ur III period (c. 2100-2000 BC)}

At some time in the Ur III period, place-value notation for sexagesimal numbers was invented (although without the use of zeros, so that the represented numbers had floating values). This is what is suggested by the existence of a number of "tables of reciprocals" from this period, with numbers in place value notation. (See Friberg 2007a, 356, and, in particular, the survey in Friberg 2009, sections 4.2.1-4, of where such tables of reciprocals have been published.) These tables of reciprocals are, by the way, the only texts from the 3rd millennium that can be said to be mathematical rather than metromathematical.

The oldest known texts with recursive algorithms are from this period. (A recursive algorithm is a procedure in several similar steps, where the result of the procedure in each step is used as data for the next step.) One such text is a calculation of the annual production of "dairy fat" and "cheese" over a period of ten years, based on the hypothetical growth of a cattle herd consisting, initially, of four milk cows. (See Nissen, Damerow, and Englund 1993, Figure 76.)

Another example is a geometrical recursive algorithm in a legal document, sworn by the name of the king, which meticulously describes how an irregularly formed "orchard" (as in Fig. 4.1 below) has been divided by parallel transversals into five smaller plots of equal areas (Clay 1915; Friberg 2009).

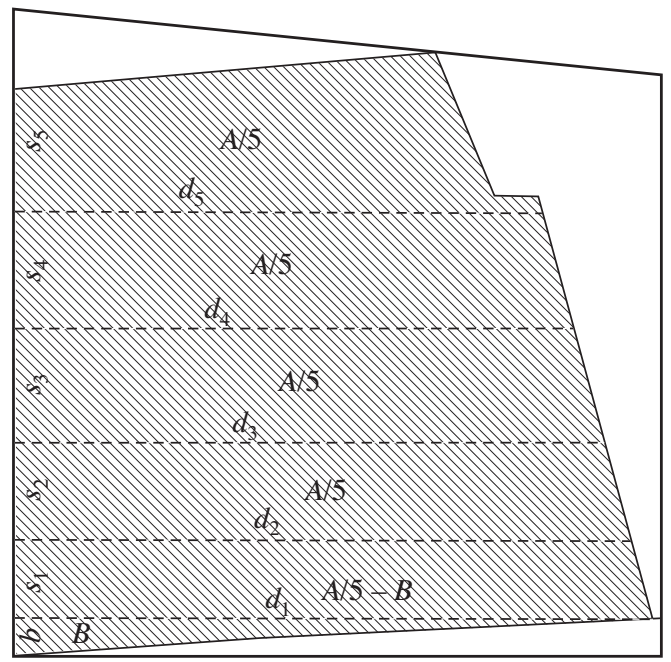

$$
\begin{aligned}
& d_{1}, f, A \text {, and } B \text { are given } \\
& \text { the sum } b+s_{1}+s_{2}+s_{3}+s_{4}+s_{5} \text { is also given } \\
& \text { sq. } d_{2}=\text { sq. } d_{1}-2 f \cdot(A / 5-B) \\
& \text { sq. } d_{n+1}=\text { sq. } d_{n}-2 f \cdot A / 5 \\
& n=2,3,4 \\
& s_{n}=1 / f \cdot\left(d_{n}-d_{n+1}\right) \\
& n=1,2,3,4
\end{aligned}
$$

Fig. 4.1. YBC 3879. The division of an orchard into five lots of equal area

The explicit computations are not shown in the text, only the results, in particular the widths of the individual plots, which are computed with surprising accuracy. The essential part of the computations must have been to divide a trapezoid of given base $d_{1}$, given growth rate, and given area, into four sub-trapezoids of given areas, proceeding as follows: First the length of the upper parallel side and the width of the first sub-trapezoid are computed. (This is a problem of the same type as the Old Akkadian trapezoid bisection problem discussed above, in section 3.3.) Then the length of the upper parallel side and the width of the second sub-trapezoid is computed. And so on. It is clear that the author of this legal text must have learned at school how to solve quadratic-linear or 
rectangular-linear problems, and also how to find approximate values for the square sides of given area numbers.

\section{Mathematical Texts from the Old Babylonian Period (c. 2000-1600 BC)}

5.1. In Old Babylonian mathematical texts, all computations are carried out in terms of abstract sexagesimal numbers in place value notation, although sometimes the given data and the answers to the problems are expressed metrologically, in terms of length and area measures, weight measures, or capacity measures. It is, therefore, quite reasonable that, as has been shown recently, the Old Babylonian mathematical school curriculum started with the copying of extensive metrological lists and tables, multiplication tables, and tables of reciprocals. (See, for instance, Proust 2007, Ch. 5.)

In the more advanced stages of the curriculum, problems involving quadratic equations of many different kinds, and many different degrees of complexity, played a central role. Another popular subject was computations with "many-place regular sexagesimal numbers". As mentioned before, a sexagesimal number $n$ is called "regular" if there exists another sexagesimal number, which conveniently may be called rec. $n$, such that $n \cdot$ rec. $n$ equals some power of 60 (= ' 1 ' in Babylonian floating place value notation). Regular sexagesimal numbers can always be interpreted as products of powers of 2,3, and 5. Well documented examples of such computations with many-place regular sexagesimal numbers are computations of square roots or reciprocals of given regular sexagesimal numbers as products of square roots or reciprocals of factors of those numbers. A particularly impressive example is the Old Babylonian text CBS 1215, which combines a "reciprocals through factorization algorithm" with a "doubling and halving algorithm". The result is a long series of pairs of reciprocals $(n$, rec. $n)$, beginning with the pair $\left(5^{3}, 5^{-3}\right)=(205,2848)$. (See Friberg 2007a, App. 3).

Interesting combinations of quadratic problems and problems involving regular sexagesimal numbers are the Old Babylonian "igi-igi.bi problems" (igi and igi.bi are Sumerian terms for $n$ and rec. $n$ ). Since, by definition, igi $\cdot$ igi.bi $=1$ (here necessarily meaning just the integer 1 ), it follows that

sq. $\{($ igi + igi.bi $) / 2\}-1=$ sq. $\{($ igi - igi.bi $) / 2\}$.

This simple identity makes it easy to solve, for instance, the quadratic equation

igi + igi.bi $=p, \quad$ with a given $p$, and with igi and igi.bi as unknowns.

Note that it also follows from the mentioned identity that if igi and igi.bi are supposed to be regular sexagesimal numbers, then

$$
(c, b, a)=((\text { igi }+ \text { igi.bi }) / 2,1,(\text { igi }- \text { igi.bi }) / 2))
$$

is a generating rule for right triangles with rational sides, "normalized" in the sense that the longest of the two orthogonal sides is of length 1. (See the detailed discussion of the famous table text Plimpton 322 (Neugebauer and Sachs 1945, text A) in Friberg 1981. The explanation proposed there of how the table on Plimpton 322 was constructed is now confirmed by a series of computations in the Old Babylonian exercises MS $3971 \S \S 3$ a-e. See Friberg 2007a, App. 8 and Sec. 10.1 f.)

5.2. Other noteworthy achievements in Old Babylonian mathematics are several types of geometric recursive algorithms. One such recursive algorithm appears on a clay tablet inscribed with the text of an exercise together with a drawing of a trapezoid divided into five sub-trapezoids, all with diagonals of length 3. (VAT 8393; see Friberg 2007b, App. 1.) The algorithm was unknown before the publication of this Old Babylonian text.

According to the statement of this problem in the cuneiform text, an initial trapezoid has a given inclined side of length $u=200$, parallel sides of given lengths $s=230$ and $t=200$, and a diagonal of length $d=300$. Asked for are two additional trapezoids "above" and two "below" the given one, all with diagonals of the same length. See Fig. 5.1 below.

In the solution in the text to this problem, a recursive algorithm is used to determine the lengths of the inclined sides and "lower" parallels of the first and second trapezoids "below" the given one, and then the lengths of the inclined sides and "upper" parallels of the first and second trapezoids "above" 
the given one. (It turns out to be impossible to continue the procedure to third trapezoids below and above the given one!)

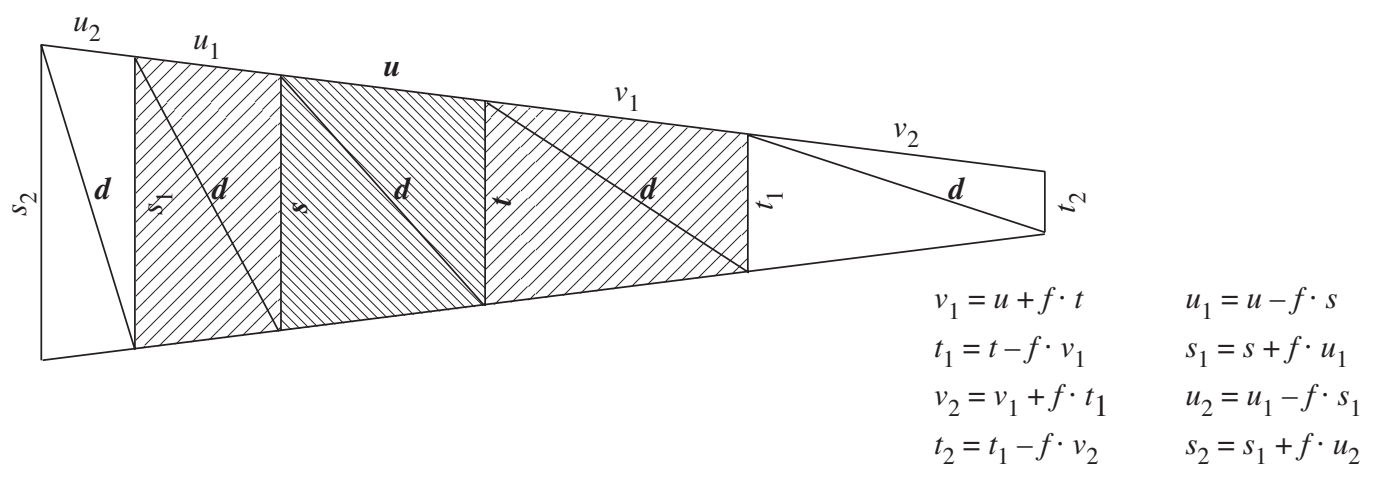

Fig. 5.1. VAT 8393. The setup for a sophisticated fixed trapezoid diagonals problem

With the given values $u=200, s=230, t=200$, and $d=300$, the values successively calculated in the algorithmic procedure of VAT 8393 are as follows (see again Fig. 5.1):

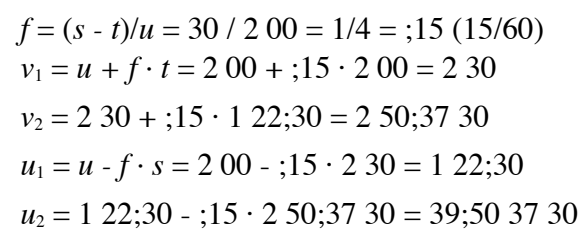

$$
\begin{aligned}
& t_{1}=t-f \cdot v_{1}=200-; 15 \cdot 230=122 ; 30 \\
& t_{2}=122 ; 30-; 15 \cdot 250 ; 3730=39 ; 503730 \\
& s_{1}=s+f \cdot u_{1}=230+; 15 \cdot 122 ; 30=250 ; 3730 \\
& s_{2}=250 ; 3730+; 15 \cdot 39 ; 503730=300 ; 35092230
\end{aligned}
$$

The procedure was probably based on geometric deliberations of the following kind: First, $a$ double application of the "rectangle diagonal rule" (a Babylonian predecessor of the so called Pythagorean theorem) was used to compute the length of the diagonal of a (symmetric) trapezoid. Namely, if $h$ is the height of the trapezoid with the parallel sides $s$ and $t$ and the inclined side $u$, then

$$
\text { sq. } u-\text { sq. }\{(s-t) / 2\}=\text { sq. } h=\text { sq. } d-\text { sq. }\{(s+t) / 2\} .
$$

Therefore, by use of the conjugate rule (see Fig. 5.2, left)

$$
\text { sq. } d=\text { sq. } u+\text { sq. }\{(s+t) / 2\}-\text { sq. }\{(s-t) / 2\}=\text { sq. } u+s \cdot t .
$$

This "symmetric trapezoid diagonal rule" is a surprising Old Babylonian precursor of Ptolemy's well known theorem about the diagonals of a cyclic quadrilateral (Heath 1981, 2:279).

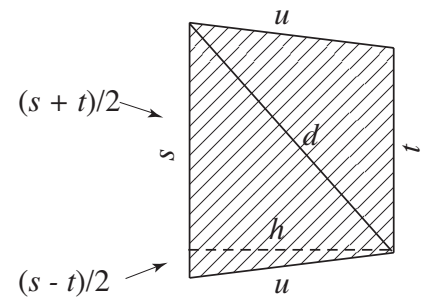

sq. $d=\mathrm{sq} \cdot u+s \cdot t$

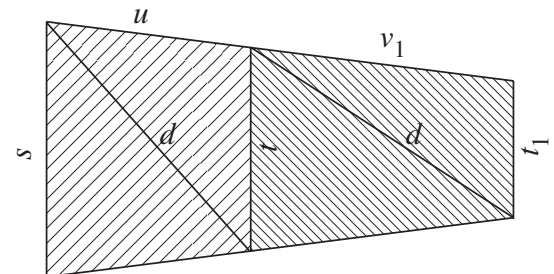

$$
v_{1}=u+f \cdot t \quad \text { and } \quad t_{1}=t-f \cdot v_{1}
$$

Fig. 5.2. Explanation of the computations in VAT 8393

Now, let

$$
f=(s-t) / u=\left(t-t_{1}\right) / v_{1}=\left(s-t_{1}\right) /\left(v_{1}+u\right)
$$

be the common growth rate of the given trapezoid and of the first trapezoid below (to the right of) it, the one with the parallel sides $t$ and $t_{1}$ and the inclined side $v_{1}$. See Fig. 5.2, right. Then $a$ double application of the symmetric trapezoid diagonal rule discussed above shows that 
sq. $u+s \cdot t=$ sq. $d=$ sq. $v_{1}+t_{1} \cdot t$.

From these equations, and from what was said about the common growth rate, it follows that

sq. $v_{1}-\mathrm{sq} . u=\left(s-t_{1}\right) \cdot t=f \cdot\left(v_{1}+u\right) \cdot t$.

Therefore, by use of the conjugate rule again,

$v_{1}-u=f \cdot t$.

Now, finally, the two equations

$v_{1}=u+f \cdot t \quad$ and $\quad t_{1}=t+f \cdot v_{1}$, where $s, t, u$, and $f$ have known values,

together yield the values of $v_{1}$ and $t_{1}$. And so on. This pair of equations deserves the name "the Old Babylonian fixed trapezoid diagonals rule". (Note that the preceding explanation of the surprisingly advanced reasoning behind the computational procedure in VAT 8393 is formally quite anachronistic, since Babylonian mathematicians had no recourse to symbolic notations.)

5.3. Just as Old Babylonian mathematicians knew a generating rule for right triangles with rational sides (or rectangles with rational sides and a rational diameter), they also knew a generating rule for divided trapezoids with rational sides and rational transversals. As shown in Fig 5.3 below, if you suppose that rational values for the partial areas and the partial lengths are known, and if you start with the three equations for the areas of the whole trapezoid and the two sub-trapezoids, you get a system of three linear equations for the unknown parallel sides, $s$ and $t$, and the unknown transversal $d$. (See the three equations in the middle of Fig. 5.3.) The solution to this system of linear equations gives corresponding rational values for $s, d$, and $t$. (See the equations to the right in Fig. 5.3.)

The resulting generating rule for a "rational trapezoid triple" $(s, d, t)$ is documented in the Old Babylonian mathematical text YBC 4608 (Neugebauer and Sachs 1945, text D; Friberg 2007b, 274) in (essentially) the following form:

$$
(s, d, t)=((P+Q) /(m+n)-Q / m+P / n, Q / m+P / n-(P+Q) /(m+n),(P+Q) /(m+n)+Q / m-P / n) .
$$

When $P=Q=B$, the same rule can be expressed in the following equivalent, but simpler, form:

$$
(s, d, t)=\{B /((m+n) \cdot m \cdot n)\} \cdot(\text { sq. } m+2 m \cdot n-\text { sq. } n \text {, sq. } m+\text { sq. } n \text {, sq. } n+2 m \cdot n-\text { sq. } m) .
$$

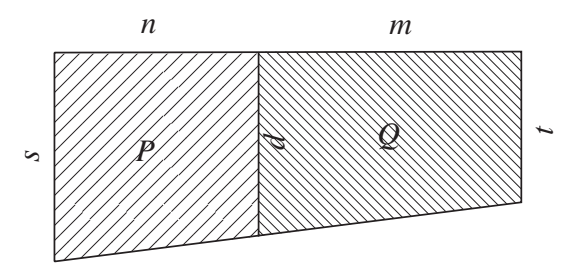

$$
\begin{array}{ll}
n, m, P \text {, and } Q \text { are given } & s+d+t=(P+Q) /(m+n)+P / n+Q / m \\
s+t=2(P+Q) /(m+n) & s=(P+Q) /(m+n)+P / n-Q / m \\
s+d=2 P / n & d=P / n+Q / m-(P+Q) /(m+n) \\
d+t=2 Q / m & t=(P+Q) /(m+n)-P / n+Q / m
\end{array}
$$

\section{Fig. 5.3. Another possible setup for a trapezoid division problem}

The mentioned generating rule must have been used also for the construction of intriguingly nice data in the Old Babylonian mathematical cuneiform text Ist. Si 269, obv. (Friberg 2007b, 279; Fig. 5.4 below). Indeed, that text contains a diagram showing four different divided trapezoids with rational sides and transversals, and with the areas of the two sub-trapezoids always in the ratio $P: Q=3000$ : $30=60: 1$ ! The sum of the lengths of the two parallel sides are always $s+t=101=60+1$ ! The sum of the partial lengths is, in all the four cases, $n+m=100=60$. The partial lengths are, in the four cases, in the ratios $n: m=15: 1,8: 1,9: 1$, and $25: 2$, respectively.

These ratios, by the way, were not randomly chosen by the author of Ist. Si 269. Indeed, it is clear from the form of the equations for $(s, d, t)$ above that for the equations to work, $n, m$, and $m+n$ must all be sexagesimal numbers! Consequently, $n / m$ and $n / m+1$ must both be regular numbers, instances of what may be called "regular twins"! True enough, the four pairs $(15,16),(8,9),(9,10)$, and $(12 ; 30$, 13;30) are all regular twins. Similarly, in YBC 4608 (above), the partial lengths $n$ and $m$ are in the ratio $1: 5$, and 1,5 , and $1+5=6$ are all regular sexagesimal numbers. Or, you can say that $(5,6)$ is another case of regular twins. 


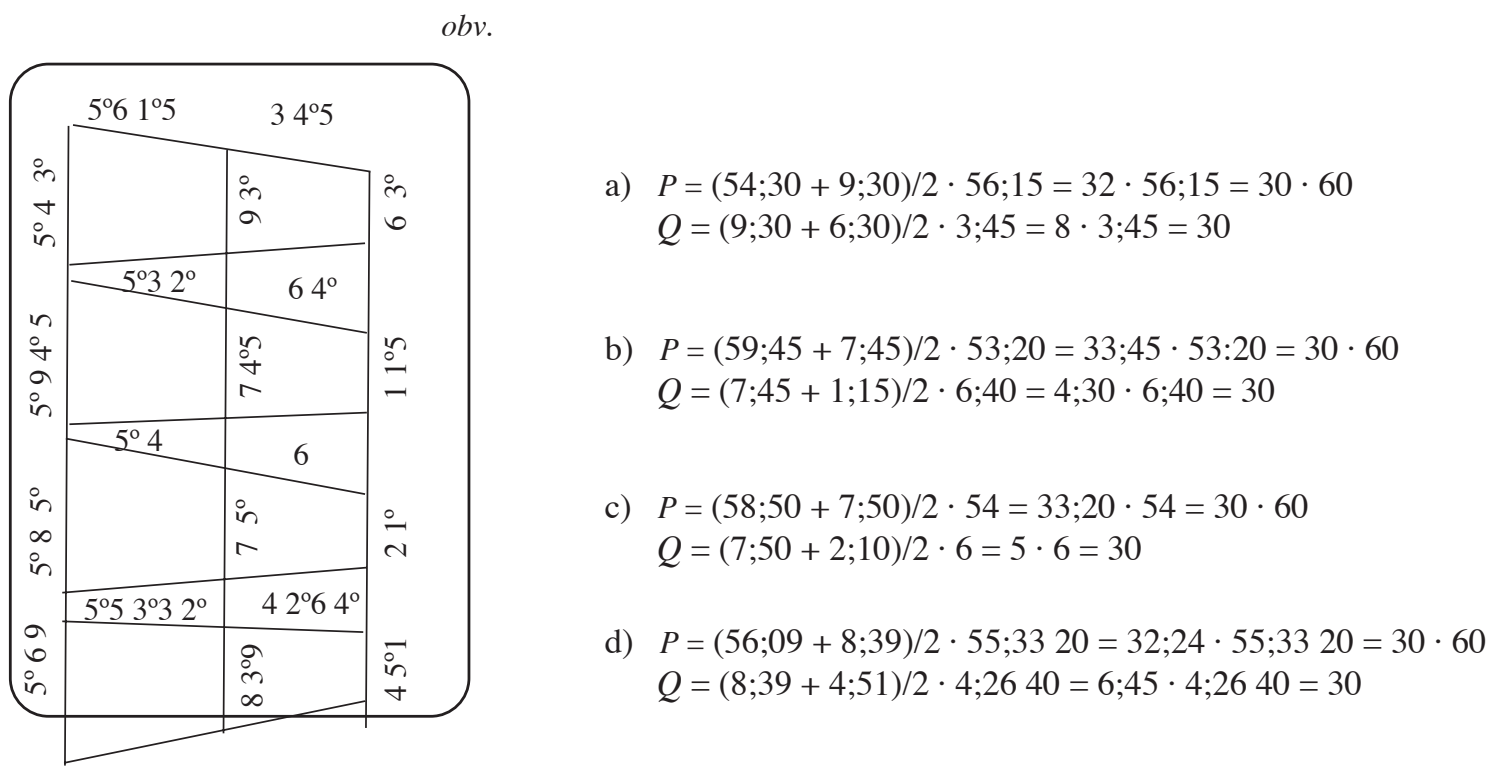

Fig. 5.4. Ist. Si 269. Four rational trapezoids with their areas divided in the ratio $60: 1$.

5.4. Just as Old Babylonian mathematicians knew that a given trapezoid with a diagonal of given length can be joined smoothly to other trapezoids with diagonals of the same length, both above and below, they also knew that a given bisected trapezoid can be joined smoothly to other bisected trapezoids, both above and below, to form a "chain of bisected trapezoids with rational sides and transversals", as in Fig. 5.5 below. (For examples, see the Old Babylonian tablets Erm. 15189 (Vaiman 1955; Friberg 2007b, 287) and AO 17264 (Neugebauer 1935, 126; Friberg 2007b, 292).)

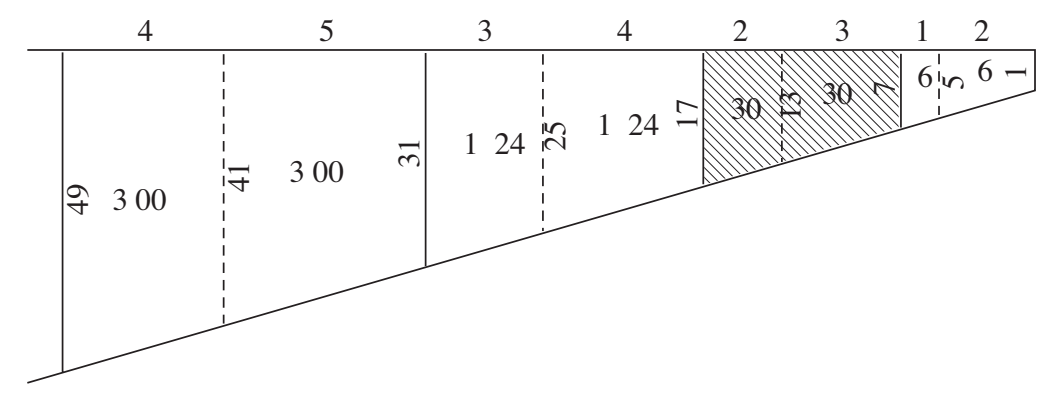

Fig. 5.5. An example of a chain of bisected trapezoids with rational sides and transversals

It is possible that Old Babylonian mathematicians found out that such a construction is possible through astute observation and experimentation, but it is also possible that they reasoned in the following way:

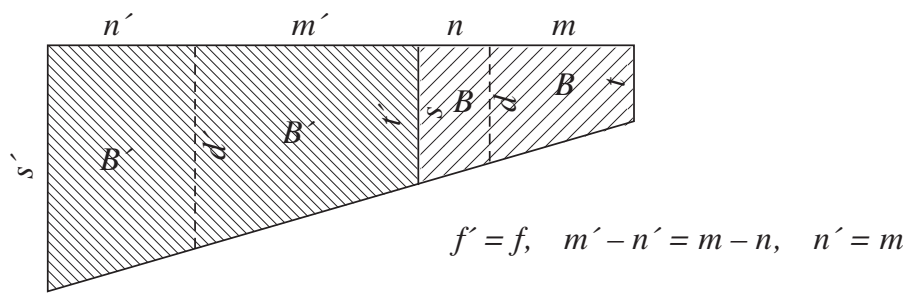

Fig. 5.6. How to join together smoothly two bisected trapezoids

In the diagram above, let $f=(s-t) /(m+n)$ be the growth rate of a given trapezoid, the one to the right in the diagram. If here $s$, and $t$ are replaced by their expressions in the Old Babylonian generating rule for trapezoid triples (see the line just above Fig. 5.3), then the equation for the growth rate takes the following new form, in terms of only the partial lengths $m, n$, and the partial area $B$ : 


$$
f=\{B /((m+n) \cdot m \cdot n)\} \cdot(2 \text { sq. } m-2 \text { sq. } n) /(m+n)=\{B /((m+n) \cdot m \cdot n)\} \cdot 2(m-n) .
$$

There is, of course, a similar expression for the trapezoid to the left. If the two trapezoids are supposed to be smoothly connected, they have a common growth rate. Therefore,

$$
\left\{B^{\prime} /\left(\left(m^{\prime}+n^{\prime}\right) \cdot m^{\prime} \cdot n^{\prime}\right)\right\} \cdot\left(m^{\prime}-n^{\prime}\right)=f^{\prime}=f=\{B /((m+n) \cdot m \cdot n)\} \cdot(m-n) .
$$

The arbitrarily imposed extra condition that

$$
m^{\prime}-n^{\prime}=m-n
$$

allows this complicated condition for a common growth rate to be simplified to

$$
B^{\prime} /\left(\left(m^{\prime}+n^{\prime}\right) \cdot m^{\prime} \cdot n^{\prime}\right)=B /((m+n) \cdot m \cdot n) .
$$

The two smoothly connected trapezoids must also have $a$ side in common, so that $t^{\prime}=s$. If also $t^{\prime}$ and $s$ are replaced by their expressions in the generating rule for trapezoid triples, it follows that

$$
\left\{B^{\prime} /\left(\left(m^{\prime}+n^{\prime}\right) \cdot m^{\prime} \cdot n^{\prime}\right)\right\} \cdot\left\{\text { sq. } n^{\prime}+2 m \cdot n-\text { sq. } m^{\prime}\right\}=t^{\prime}=s=\{B /((m+n) \cdot m \cdot n)\} \cdot\{\text { sq. } m+2 m \cdot n-\text { sq. } n\} .
$$

In view of the condition for a common growth rate, this condition can be simplified to

$$
\text { sq. } n^{\prime}+2 m \cdot n-\text { sq. } m^{\prime}=\text { sq. } m+2 m \cdot n-\text { sq. } n \text {, }
$$

or, equivalently,

$$
2 \text { sq. } n^{\prime}-\text { sq. }\left(m^{\prime}-n^{\prime}\right)=2 \text { sq. } m-\text { sq. }(m-n) .
$$

In view of the extra condition that $m^{\prime}-n^{\prime}=m-n$, this equation, in its turn, can be simplified to

$$
2 \text { sq. } n^{\prime}=2 \text { sq. } m \text {, or simply } n^{\prime}=m \text {. }
$$

Summing up, one finds that a sufficient, but perhaps not necessary, condition for the two bisected trapezoids in the figure above to be smoothly connected is that

$$
f^{\prime}=f, \quad m^{\prime}-n^{\prime}=m-n, \quad \text { and } \quad n^{\prime}=m .
$$

It is, indeed, easy to see that these conditions are satisfied in the numerical example shown above, in Fig. 5.5. It is also easy to see that if the numerical data for one bisected trapezoid are given, then the corresponding numerical data for smoothly connected bisected trapezoids above and below the given one can be computed, effortlessly, by use of these equations alone. Therefore these three conditions together may be called a generating rule for a "chain of (rational) bisected trapezoids".

5.5. In the Old Babylonian mathematical cuneiform texts TMS 23 (Bruins and Rutten 1961; Friberg 2007b, 300) and Erm. 15073 (Vaiman 1961; Friberg 2007b, 304), the idea of what may be called "confluent trapezoid bisections" is behind two different, but equivalent, generating rules, by use of which it is possible to construct any number of rational sub-trapezoids of a given rational bisected trapezoid, all bisected by the same transversal.

The first of these generating rules can be explained as follows: In Fig. 5.7 below, suppose that the given bisected trapezoid has parallel sides of lengths $s$ and $t$, and let $p$ and $q$ be the heights of two subtrapezoids cut off at each end of the given trapezoid. Suppose that what then remains of the original bisected trapezoid is a bisected sub-trapezoid with the same bisecting transversal and with parallel sides of lengths $s^{\prime}$ and $t^{\prime}$.

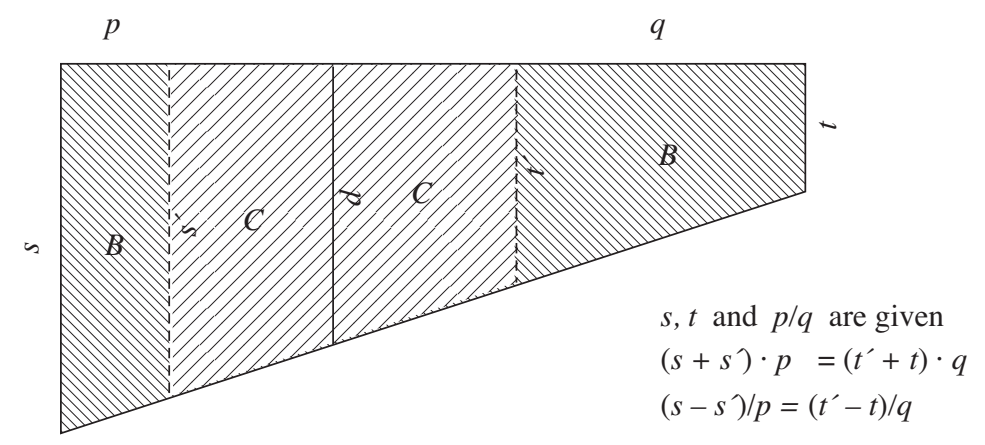

Fig. 5.7. How to construct confluent trapezoid bisections 
The two cut-off trapezoids then obviously have equal areas and equal growth rates. More precisely, in modern symbolic notations,

$$
\left(s+s^{\prime}\right) \cdot p=\left(t+t^{\prime}\right) \cdot q \text { and }\left(s-s^{\prime}\right) / p=\left(t^{\prime}-t\right) / q .
$$

This is a system of two linear equations for the two unknowns $s^{\prime}$ and $t^{\prime}$. The solution, again in modern symbolic notations, can be shown to be of the form

$$
s^{\prime}=a \cdot s+b \cdot t, \quad t^{\prime}=b \cdot s-a \cdot t,
$$

where

$$
a=2 q \cdot p /(\text { sq. } q+\text { sq. } p) \quad \text { and } \quad b=(\text { sq. } q-\text { sq. } p) /(\text { sq. } q+\text { sq. } p) .
$$

It is easy to see that the expressions above for $a$ and $b$, with $p$ and $q$ rational, can be interpreted as a generating rule for the orthogonal sides of rational right triangles with diagonals of length 1 .

In other words, the first of the Old Babylonian rules for the construction of confluent bisected trapezoids corresponds to a composition of rational solutions to two kinds of indeterminate quadratic equations, the "trapezoid bisection equation" and the "rectangle diagonal equation", namely

sq. $s+$ sq. $t=2$ sq. $d$ and $\quad$ sq. $a+$ sq. $b=$ sq. $c$ (in the case when $c=1$ ).

The underlying observation is the following "composition rule":

sq. $(a \cdot s+b \cdot t)+$ sq. $(b \cdot s-a \cdot t)=($ sq. $a+$ sq. $b) \cdot($ sq. $s+$ sq. $t)$.

See the extended historical discussion of this composition rule in Friberg 2007b, $345 \mathrm{ff}$.

5.6. The second rule for the construction of confluent bisected trapezoids used in the Old Babylonian problem text TMS 23 can be explained as follows (Friberg 2007b, 300). Let $f$ be the growth rate of the given trapezoid in Fig. 5.7 above. Then,

$$
s^{\prime}=s-f \cdot p, \quad t^{\prime}=t+f \cdot q \quad \text { and } \quad(2 s-f \cdot p) \cdot p=(2 t+f \cdot q) \cdot q(=2 B) .
$$

From the last of these equations it follows that the value of $f$ can be computed as follows:

$$
f=(s \cdot p-t \cdot q) /\{(\text { sq. } p+\text { sq. } q) / 2\} .
$$

Consequently, the second rule for the construction of confluent bisected trapezoids is simply

$$
\left.s^{\prime}=s-p \cdot(s \cdot p-t \cdot q) /\{(\text { sq. } p+\text { sq. } q) / 2\}, \quad \text { and } \quad t^{\prime}=t+q \cdot(s \cdot p-t \cdot q) /\{\text { (sq. } p+\text { sq. } q) / 2\right\} .
$$

In the application of the first of the two rules for the construction of confluent bisected trapezoids in the mentioned Old Babylonian problem text TMS 23, the parameters used are, apparently, the following ones:

$$
\begin{aligned}
& s=145(=15 \cdot 7), \quad t=15(=15 \cdot 1), \quad \text { and } \quad p / q=1 / 3, \text { so that } \\
& (\text { sq. } 3 \text { - sq. } 1) /(\text { sq. } 3+\text { sq. } 1)=4 / 5=; 48, \quad \text { and } \quad(2 \cdot 3 \cdot 1) /(\text { sq. } 3+\text { sq. } 1)=3 / 5=; 36 .
\end{aligned}
$$

Accordingly, in this example the lengths of the parallel sides of the sub-trapezoid were computed as

$$
s^{\prime}=; 48 \cdot 145+; 36 \cdot 15=124+9=133(=3 \cdot 31), \quad t^{\prime}=; 36 \cdot 145-; 48 \cdot 15=103-12=51(=3 \cdot 17) .
$$

The solution is correct, which can be shown as follows:

$$
\begin{aligned}
& (\text { sq. } s+\text { sq. } t) / 2=(\text { sq. } 145+\text { sq. } 15) / 2=(30345+345) / 2=30730 / 2=13345=\text { sq. } 115, \quad d=115(=3 \cdot 25), \\
& \left(\text { sq. } s^{\prime}+\text { sq. } t^{\prime}\right) / 2=(\text { sq. } 133+\text { sq. } 51) / 2=(22409+4321) / 2=30730 / 2=13345=\text { sq. } 115, \quad d^{\prime}=115=d .
\end{aligned}
$$

(Note that the triples $(s, d, t)=(1,5,7)$ and $(s, d, t)=(17,25,31)$ both can be found in the diagram in Fig. 5.5 above.)

In the application of the second rule in the same cuneiform text, TMS 23, the parameters used are $s=125(=5 \cdot 17), \quad t=35(=5 \cdot 7), \quad$ and $\quad p / q=1 / 2$.

Accordingly, the lengths of the parallel sides of the sub-trapezoid were computed as

$$
\begin{aligned}
& \left.s^{\prime}=125-1 \cdot(125 \cdot 1-35 \cdot 2) /\{\text { (sq. } 2+\text { sq. } 1) / 2\right\}=125-1 \cdot 6=119, \quad \text { and } \\
& t^{\prime}=35+2 \cdot(125 \cdot 1-35 \cdot 2) /\{(\text { sq. } 2+\text { sq. } 1) / 2\}=35+2 \cdot 6=47 .
\end{aligned}
$$

The solution is correct, which can be shown as follows:

$($ sq. $s+$ sq. $t) / 2=($ sq. $125+$ sq. 35$) / 2=(20025+2025) / 2=22050 / 2=11025=$ sq. $105, \quad d=105(=5 \cdot 13)$, 


$$
\left(\text { sq. } s^{\prime}+\text { sq. } t^{\prime}\right) / 2=(\text { sq. } 119+\text { sq. } 47) / 2=(14401+3649) / 2=22050 / 2=11025=\text { sq. } 105, \quad d^{\prime}=105=d \text {. }
$$

Note, by the way, that the triple $(s, d, t)=(7,13,17)$ but not the triple $(s, d, t)=(47,105,119)$ can be found in the diagram in Fig. 5.5 above. This implies that there must exist other possible chains of bisected trapezoids with rational sides than the one shown in Fig. 5.5, for instance the one below:

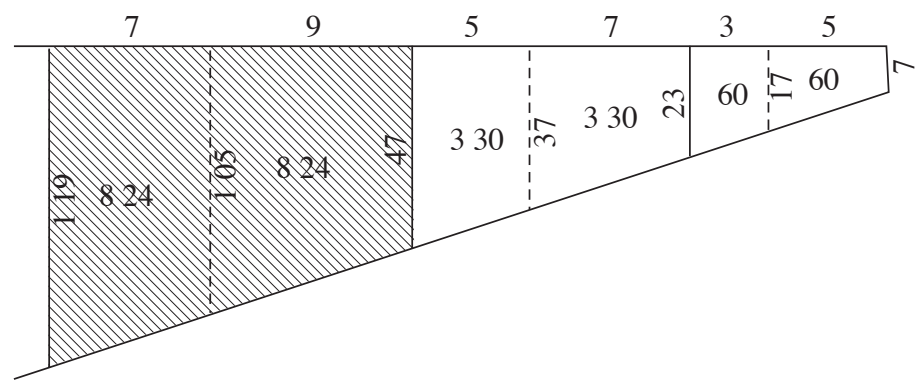

Fig. 5.8. Another example of a chain of bisected trapezoids with rational sides and transversals

\section{Mathematical Texts from the Kassite Period (2nd Half of 2nd Millennium BC)}

6.1. The few mathematical problem texts from the Kassite period that are known are similar to Old Babylonian problem texts, but are, for some reason, exceptionally sophisticated. One such text contains the computation of the weight of a colossal icosahedron made of 20 equilateral copper triangles (Friberg 2007a, 342).

Another such text is a single problem text containing a kind of "boundary value problem" for a trapezoid with sides of given lengths, divided into a chain of three bisected trapezoids (AO 17264; Neugebauer 1935, 126; Friberg 2007b, 292). The stated problem is to find out how the given trapezoid can be divided among six brothers so that the oldest brother and the next one get trapezoidal shares of the same area, so that numbers 3 and 4 get the same, and also numbers 5 and 6. See Fig. 6.1 below. The student who wrote down his solution to the problem cheated blatantly, but managed to find the correct result (which he obviously knew beforehand) through a couple of nonsense calculations.

It is likely that the solution to the mentioned problem was meant to proceed in the following way. In the figure below of the divided trapezoid (Fig. 6.1), the lengths $s^{\prime}$ and $t^{\prime \prime}$ of the outermost parallels and the length $u$ of the height are supposed to be known. Also known is, consequently, the growth rate

$$
f=\left(s^{\prime}-t^{\prime \prime}\right) / u \text {. }
$$

Now, suppose (in order to make the situation as symmetric as possible) that the chain of three bisected trapezoids in the figure below has been constructed with departure from the bisected trapezoid in the middle, with the (unknown) parameters

$n, m, s$, and $t$,

and that the added bisected trapezoids to the left and right have been constructed by use of the generating rule for a chain of bisected trapezoids. See section 5.4 above. Then, as indicated in Fig. 6.1 below, the corresponding parameters for the trapezoid to the left are

$m, 2 m-n, s^{\prime}$, and $s$,

while the parameters for the trapezoid to the right are

$2 n-m, n, t$ and $t^{\prime \prime}$.

The growth rate $f$ is known. Therefore, if only the values of $m$ and $n$, too, can be computed in some way, it will be easy to compute, subsequently, also the lengths of all the parallel lines in Fig. 6.1.

Now, one equation for $m$ and $n$ is obvious, namely

$3(m+n)=m+(2 m-n)+n+m+(2 n-m)+n=u$.

(The cheating student managed to get this equation right, at least!) 


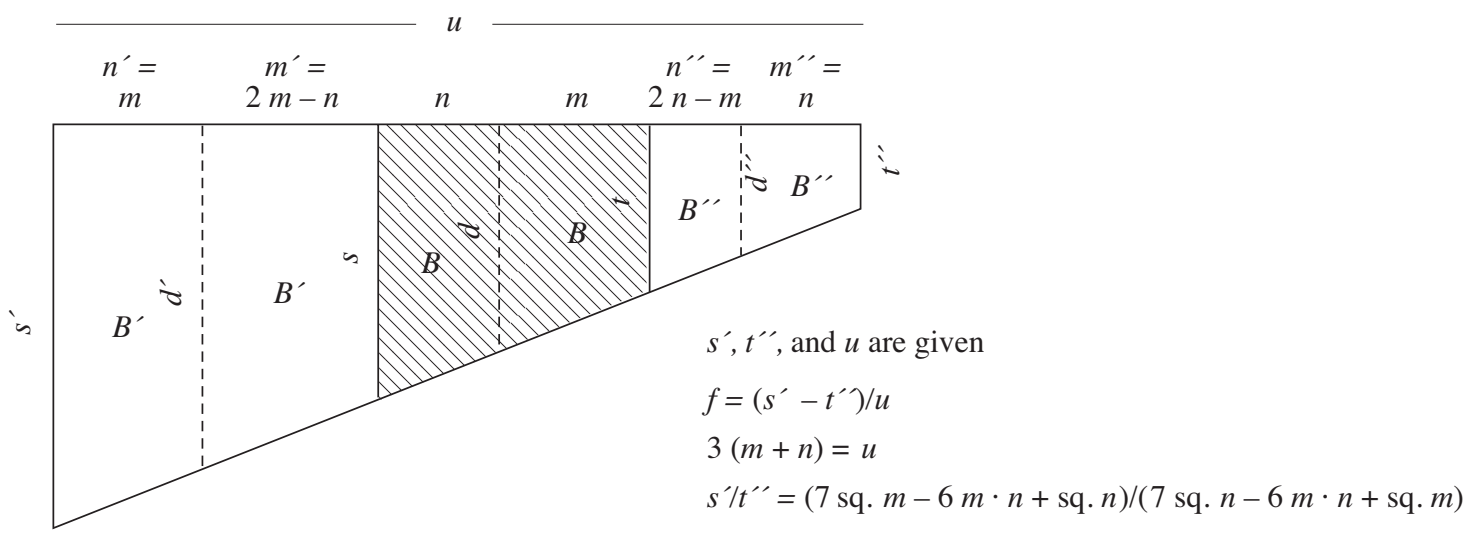

Fig. 6.1. AO 17264. Dividing a trapezoidal field between six brothers

Another equation for $m$ and $n$ is a consequence of the generating rule for bisected trapezoids If the rule is used on the trapezoid to the left in Fig. 6.1, one sees that

$s^{\prime} / t^{\prime \prime}=\left(\right.$ sq. $m^{\prime}+2 m^{\prime} \cdot n^{\prime}-$ sq. $\left.n^{\prime}\right) /($ sq. $(2 n-m)+2 n \cdot(2 n-m)-$ sq. $n)$.

Clearly, now

sq. $m^{\prime}+2 m^{\prime} \cdot n^{\prime}-$ sq. $n^{\prime}=$ sq. $(2 m-n)+2(2 m-n) \cdot m-$ sq. $m=7$ sq. $m-6 m \cdot n+$ sq. $n$.

Similarly,

sq. $(2 n-m)+2 n \cdot(2 n-m)-$ sq. $n=7$ sq. $n-6 m \cdot n+$ sq. $m$.

On the other hand, both $s^{\prime}$ and $t^{\prime \prime}$ have given values. This means that $m$ and $n$ are connected to each other by the following quadratic-linear system of equations:

$s^{\prime} / t^{\prime \prime}=(7 \mathrm{sq} . m-6 m \cdot n+$ sq. $n) /(7$ sq. $n-6 m \cdot n+$ sq. $m), \quad m+n=u / 3$.

The given parameters in the mentioned Kassite problem text AO 17264 are

$s^{\prime}=333, \quad t^{\prime \prime}=51, \quad u=215$.

Therefore, in that particular case, $m$ and $n$ are solutions to the equations:

$333 \cdot(7 \mathrm{sq} . n-6 m \cdot n+\mathrm{sq} . m)=51 \cdot(7 \mathrm{sq} . m-6 m \cdot n+$ sq. $n), \quad m+n=45$.

After simplification, this becomes the following quadratic-linear system of equations

4 sq. $m+27 m \cdot n=40$ sq. $n, \quad m+n=45$.

Because the quadratic equation is homogeneous (it has no linear term), it can be solved in the following way: Suppose that

$m=p \cdot n, \quad$ where $p$ is a new unknown.

Then, after elimination of a factor sq. $n$,

4 sq. $p+27 p=40$.

This quadratic equation has the solution $p=5 / 4=1 ; 15$. Consequently, the original quadratic-linear system of equations can be replaced by the following system of only linear equations:

$m=1 ; 15 \cdot n, \quad m+n=45$.

It is easy to see that the solution to this system of linear equations is

$m=25, \quad n=20$.

Consequently, the 5 transversals in the divided trapezoid in Fig. 6.1 divide the height of the trapezoid into the following 6 partial lengths:

$m, 2 m-n, n, m, 2 n-m, n=25,30,20,25,15,20$.

On the other hand,

$f=(333-51) / 215=242 / 215=6 / 5=1 ; 12$.

Therefore,

$d^{\prime}=s^{\prime}-f \cdot m=333-1 ; 12 \cdot 25=333-30=303$, 
and so on. The complete solution is

$$
s^{\prime}, d^{\prime}, s, d, t, d^{\prime \prime}, t^{\prime \prime}=333,303,227,203,133,115,51=3 \cdot(111,101,49,41,31,25,17) .
$$

(Compare this result with the chain of trapezoids in Fig. 5.5 above.)

Thus, although the student who wrote the text AO 17264 found the correct solution by cheating and knowing the answer beforehand, he should have been able to find the solution honestly by working hard in the way described above.

6.2. It is instructive to see how the method used above to solve the problem stated in AO 17264 would work in a related but (much) simpler case. (This particular case is undocumented, but it is not uncommon in Babylonian mathematical texts that a given type of problem is worked through in a number of related cases, usually starting with the simplest possible case.) Suppose, therefore, that a trapezoidal field with the length $100(=60)$ and with parallel sides of lengths 7 and 31 shall be divided between four brothers, so that the oldest brother and the next brother get trapezoidal shares of the same size, and so that also brothers 3 and 4 get equal trapezoidal shares.

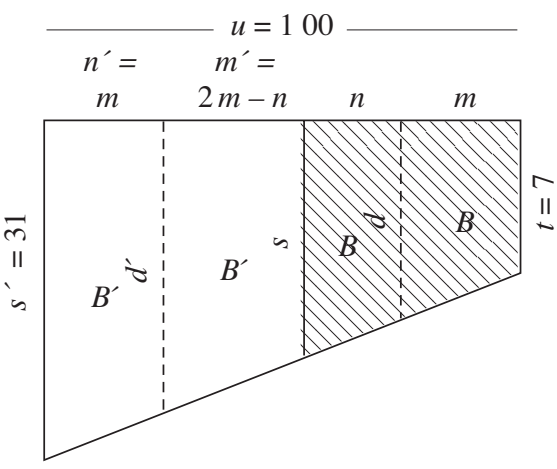

$$
\begin{aligned}
& s^{\prime}=31, t=7, \text { and } u=100 \text { are given } \\
& f=\left(s^{\prime}-t\right) / u \\
& 4 m=u \\
& s^{\prime} / t=(7 \mathrm{sq} \cdot m-6 m \cdot n+\text { sq. } n) /(\text { sq. } n+2 m \cdot n-\text { sq. } m)
\end{aligned}
$$

Fig. 6.2. Dividing a trapezoidal field between four brothers

The new situation is described in Fig. 6.2. above. In this situation,

$$
u=m+(2 m-n)+n+m=4 m \text {, so that } m=u / 4=15 \text {. }
$$

In addition, it follows from the generating rule for a chain of bisected trapezoids (see section 5.4) that (7 sq. $m-6 m \cdot n+$ sq. $n) /($ sq. $n+2 m \cdot n-$ sq. $m)=s^{\prime} / t=31 / 7$.

Expressed differently,

$$
7 \cdot(7 \mathrm{sq} . m-6 m \cdot n+\mathrm{sq} . n)=31 \cdot(\text { sq. } n+2 m \cdot n-\text { sq. } m) \text {. }
$$

After simplification, this equation is replaced by the new equation

$$
10 \text { sq. } m=3 \text { sq. } n+13 m \cdot n \text {. }
$$

Since $m=15$ is already known, this is a quadratic equation for $n$ :

$10 \cdot 345=3$ sq. $n+13 \cdot 15 \cdot n$, or, after division by 3 , sq. $n+105 n=1230$.

Solving this quadratic equation, one finds that $n=10$. Consequently, $m, 2 m-n, n, m=15,20,10,15, \quad$ and $\quad s^{\prime}, d^{\prime}, s, d, t=31,25,17,13,7$.

This result agrees with what is shown in Fig. 5.5 above.

\section{Mathematical Texts from the Achaemenid Perid (c. 540-330 BC)}

There are only a few known mathematical cuneiform texts from this period, among other things for the reason that clay tablets as a medium for writing in cuneiform had largely fallen out of use, and the new writing materials were far less durable than clay and have not been preserved. Nevertheless, the few still existing Achaemenid mathematical cuneiform texts show quite clearly that not all of the Old Babylonian achievements in mathematics had been forgotten a whole millennium after the end of the Old Babylonian period.

Thus, the Old Babylonian factorization method for the computation of reciprocals of regular 
sexagesimal numbers by use of clever arithmetical algorithms was still in use, while the standard Old Babylonian 2-place table of reciprocals had grown into much larger "many-place" tables. (See, for instance, Friberg 1999b). Quadratic equations were still being studied, in particular the mentioned "igi-igi.bi problems" (see section 5.1 above), which are closely related to the generating rules for right triangles with rational sides. The affinity between Old Babylonian and Achaemenid mathematical texts is even more clear in view of the fact that in some known Achaemenid mathematical texts (W 23291 and W 23291-x) mathematical problems have a solution in terms of Old Babylonian length and surface measures, side by side with a solution in terms of contemporary length and surface measures. (See Friberg et al. 1990, §§ 4-8 and Friberg 1997, §§ 1-3.)

There is only one example of a geometrical recursive algorithm in the known Achaemenid mathematical texts, but that example appears twice, in one case in an "Old Babylonian" version, in another case in a contemporary version. In the Old Babylonian version (Fig. 7.1 below; Friberg, et al. 1990, 494), the stated problem is how a circle of given circumference 60 rods can be divided into five concentric circular rings, all of the same given width, 2 rods. In the Achaemenid version (W 23291, § 1g; Friberg 1997, 272), the question is how a circle of given "seed (surface) measure" can be divided into concentric circular rings, all of the given width 1 rod.

The problem in W 23291-x, $\S 2$ is solved in the following way. Let $A$ be the area of a circle, $a$ its circumference, and $d$ its diameter. Then in this text, as normally also in Old Babylonian mathematical texts, the following simple but convenient assumptions are made:

$$
A=\text { (appr.) ;05 · sq. } a, \quad d=\text { (appr.) ;20 } a \text {. }
$$

As a consequence of these assumptions, the areas and circumferences of the concentric circles in the figure below can be computed by use of the following recursive algorithms:

$$
\begin{aligned}
& a_{1}=1(60), \quad d_{1}=20, \quad A_{1}=500, \\
& d_{n}-d_{n+1}=2 \cdot 2, \quad a_{n}-a_{n+1}=3 \cdot 2 \cdot 2, \quad A_{n}-A_{n+1}=\left(a_{n}+a_{n+1}\right) \cdot 1 \quad \text { for } n=1,2,3,4 .
\end{aligned}
$$

The result of the computations is indicated in a diagram in the cuneiform text, namely that the five circumferences are $1(00), 48,36,24$, and 12 (rods), that the five diameters are $20,16,12,8$, and 4 (rods), and that the areas of the five circular bands are 148,1 24, 1 (00), 36, and 12 (square rods).

The algorithmic calculation in the text proceeds, essentially, in the following series of steps:

$$
\begin{aligned}
& a_{2}=100-3 \cdot 2 \cdot 2=48, \quad A_{1}-A_{2}=(100+48) / 2 \cdot 2=54 \cdot 2=148, \\
& a_{3}=48-3 \cdot 2 \cdot 2=36, \quad A_{2}-A_{3}=(48+36) / 2 \cdot 2=42 \cdot 2=124 \\
& a_{4}=36-3 \cdot 2 \cdot 2=24, \quad A_{3}-A_{4}=(36+24) / 2 \cdot 2=30 \cdot 2=100, \\
& a_{5}=24-3 \cdot 2 \cdot 2=12, \quad A_{4}-A_{5}=(24+12) / 2 \cdot 2=18 \cdot 2=36, \\
& A_{5}=12 \cdot 12 \cdot ; 05=12, \quad\left(A_{1}-A_{2}\right)+\left(A_{2}-A_{3}\right)+\left(A_{3}-A_{4}\right)+\left(A_{4}-A_{5}\right)+A_{5}=148+124+100+36+12=500=A_{1} .
\end{aligned}
$$

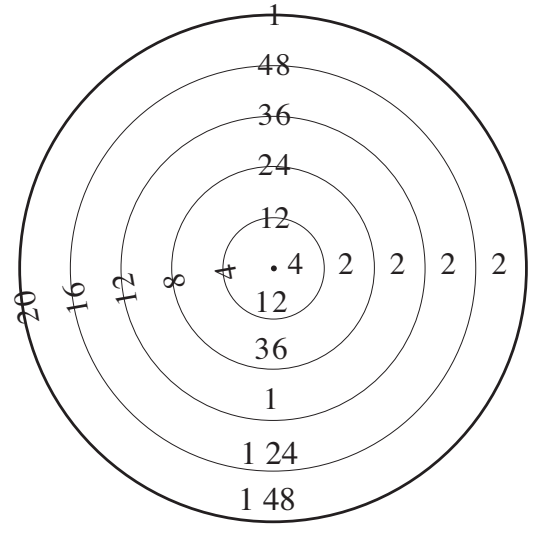

$$
\begin{aligned}
& \text { given circumference: } \quad a_{1}=100 \\
& \text { given constant width: } \quad w=2 \\
& \text { diameter: } \quad d_{1}=; 20 \cdot a_{1}=20 \\
& \text { area: } \quad A_{1}=; 05 \cdot \text { sq. } a_{1}=500 \\
& d_{n}-d_{n+1}=2 \cdot w=2 \cdot 2 \quad n=1,2,3,4,5 \\
& a_{n}-a_{n+1}=3 \cdot 2 \cdot 2, \quad n=1,2,3,4,5 \\
& A_{n}-A_{n+1}=\left(\text { sq. } a_{n}-\text { sq. } a_{n+1}\right) / 12 \\
& =\left(a_{n}+a_{n+1}\right) / 2 \cdot\left(a_{n}-a_{n+1}\right) / 6 \\
& =\left(a_{n}+a_{n+1}\right) / 2 \cdot 2 \\
& n=1,2,3,4
\end{aligned}
$$

Fig. 7.1. W 23291-x, § 2. Division of a circle into five circular rings of equal width 


\section{Conclusion}

The author's intention has been that the discussion above should demonstrate the amazing persistence of the Mesopotamian mathematical tradition, stretching over three full millennia and many different historical periods, and involving a long series of impressive developments of mathematical ideas and methods. One clear but unsurprising example of the longevity of Mesopotamian mathematical traditions is the continued use of sexagesimal numbers as the numbers used for calculations, from, probably, before the invention of writing in the 4th millennium BC and for as long as the cuneiform script stayed in use. Interesting mathematical problems in terms of regular sexagesimal numbers, often many-place such numbers, appear first in texts from the Old Akkadian period in the 3rd millennium BC, but enjoyed a great popularity also in mathematical cuneiform texts from the Old Babylonian period in the 2nd millennium BC, and from the Achaemenid and later periods in the 1 st millennium BC.

The real surprise is the discovery of the central role played by various methods of formulating and solving more or less difficult field division problems, beginning with the field expansion procedure, which appears to have been in use for about a millennium with start in the proto-literate period, until it was replaced by more exact methods to solve quadratic equations, probably in the Old Akkadian period. In the more advanced stages of the curriculum of the Old Babylonian scribe school, the study of problems involving quadratic equations of many different kinds, and many different degrees of complexity, played a central role (as shown in Høyrup 2002). However, the most impressive results of this study was a truly astonishing variety of recursive geometric algorithms for the repeated division of trapezoidal fields into chains of sub-trapezoids with rational sides and transversals or diagonals. Even after the intense blossoming of mathematics in the Old Babylonian period, the interest for imaginative field division problems remained, which is shown by interesting trapezoid division and circle division texts from the Kassite and Achaemenid periods.

Incidentally, the last blossoming of Mesopotamian mathematics was the amazing rise of mathematical astronomy in the second half of the 1st millennium BC. This development would scarcely have been possible without, for instance, the previous invention of sexagesimal numbers in place value notation, or operations with with many-place regular sexagesimal numbers using clever arithmetical algorithms, or the intensive occupation with geometrical recursive algorithms.

It ought to be evident that so many achievements in Mesopotamian mathematics over such a long period of time could not pass unnoticed by surrounding cultures, even if, by necessity, the detailed mechanisms of any diffusion of knowledge will remain unknown. Anyway, in the 18 chapters of Friberg 2007b, it is shown by a multitude of elaborately argued examples that many of the basic concepts and methods in classical Greek mathematics have obvious Babylonian predecessors. Among those important parts of Greek mathematics with Babylonian predecessors are the quadratic equations in Books II and X of Euclid's Elements, in Euclid's Data, and in Diophantus' Arithmetica (Friberg 2007b, Chs. 1, 5, 10, and 13), and the division of figures in Euclid's lost book On Divisions (op. cit., Ch. 11).

A complement to Friberg 2007b is, by the way, Friberg 2013, where it is shown that also classical Greek music theory in works by Euclid and Ptolemy, and others, in particular the construction of diatonic heptatonic scales by use of recursive tuning procedures, had important Old Babylonian predecessors. Interestingly, in one Old Babylonian text, the seven notes of a heptatonic scale, and certain intervals between them, were represented geometrically by the points and sides of a seven-sided star figure. 


\section{References}

Bruins, E. M., Rutten, M. (1961) Textes mathématiques de Suse. Paris: Paul Geuthner.

Clay, A. T. (1915) Miscellaneous Inscriptions in the Yale Babylonian Collection (YOS 1). New Haven: Yale University Press.

Deimel, A. (1923) Schultexte aus Fara (WVDOG 43). Leipzig.

Englund, R. K., Grégoire, J.-P. (1991) The Proto-Cuneiform Texts from Jemdet Nasr (MSVO 1). Berlin: Gebr. Mann.

Englund, R. K. (1997) Proto-Cuneiform Texts from Diverse Collections (MSVO 4). Berlin: Gebr. Mann.

Friberg, J. (1981) "Plimpton 322, Pythagorean triples, and the Babylonian triangle parameter equations", Historia Mathematica 8, 277-318.

(1986) "Three remarkable texts from ancient Ebla", Vicino Oriente 6, 3-25.

- , H. Hunger, F. N. Al-Rawi (1990) “'Seed and Reeds', a metro-mathematical topic text from Late Babylonian Uruk", Baghdader Mitteilungen 21, 483-557, pl. 46-48.

(1997) "'Seed and Reeds Continued'. Another metro-mathematical topic text from Late Babylonian Uruk", Baghdader Mitteilungen 28, 251-365, pl. 45-46.

(1997/98) "Round and almost round numbers in proto-literate metro-mathematical field texts", Archiv für Orientforschung 44/45, 1-58.

(1999a) "Proto-literate counting and accounting in the Middle East. Examples from two new volumes of proto-cuneiform texts", Journal of Cuneiform Studies 51, 107-137.

(1999b) "A Late Babylonian factorization algorithm for the computation of reciprocals of many-place sexagesimal number", Baghdader Mitteilungen 30, 139-161, 2 pl.

(2005) Unexpected Links between Egyptian and Babylonian Mathematics. Singapore: WorldScientific.

(2007a) A Remarkable Collection of Babylonian Mathematical Texts. (Manuscripts in the Schøyen Collection: Cuneiform Texts 1). New York: Springer.

(2007b) Amazing Traces of a Babylonian Origin in Greek Mathematics. Singapore: WorldScientific.

(2008) "A remarkable collection of Babylonian mathematical texts", Notices of the American Mathematical Society 55, 1076-87.

(2009) "A geometric algorithm with solutions to quadratic equations in a Sumerian Juridical document from Ur III Umma", Cuneiform Digital Library Journal 2009:3.

(2013) "Seven-sided star figures and tuning algorithms in Mesopotamian, Greek, and Islamic texts", Archiv für Orientforschung 52, 121-155.

Green, M. W., Nissen, H. J. (1987) Zeichenliste der archaischen Texte aus Uruk (ATU 2). Berlin: Gebr. Mann.

Heath, T. L. (1981) A History of Greek Mathematics, vol. 2. New York: Dover Publications.

Høyrup, J. (1982) "Investigations of an Early Sumerian division problem", Historia Mathematica 9, 19-36.

(1996) "Changing trends in the historiography of Mesopotamian mathematics: An insider's view". History of Science 34, 1-32.

(2002) Lengths, Widths, Surfaces: A Portrait of Old Babylonian Algebra and its Kin. New 
York: Springer.

Jestin, R. R. (1937) Tablettes sumériennes de Shuruppak. Paris.

Luckenbill, D. D. (1930) Inscriptions from Adab (OIP 14). Chicago.

Neugebauer, O. (1935) Matematische Keilschrift-Texte 1. Berlin: Springer Verlag. Society.

Nissen, H. J., P. Damerow, and R.K. Englund (1993). Archaic Bookkeeping, Early Writing and Techniques of Economic Administration in the Ancient Near East. Chicago, London: The University of Chicago Press.

Proust, C. (2007) Tablettes mathématiques de Nippur. Paris: De Boccard.

(2008) Tablettes mathématiques de la collection Hilprecht. Wiesbaden: Harassowitz.

Vaiman, A. A. (1955) "Ermitazhnaya klinopisnaya matematicheskaya tablichka No. 015189", Epigrafika Vostoka 10: 71-83, Pl. 1-2.

- (1961) Shumero-vavilonskaya matematika. Moskva: Izdatel'stvo vostochnoi literatury. 